\title{
Transducer modeling for accurate acoustic simulations of transcranial focused ultrasound stimulation
}

Pasquinelli, Cristina; Montanaro, Hazael; Lee, Hyunjoo J.; Hanson, Lars G.; Kim, Hyungkook; Kuster, Niels; Siebner, Hartwig R.; Neufeld, Esra; Thielscher, Axel

Published in:

Journal of Neural Engineering

Link to article, DOI:

$10.1088 / 1741-2552 / a b 98 d c$

Publication date:

2020

Document Version

Peer reviewed version

Link back to DTU Orbit

Citation (APA):

Pasquinelli, C., Montanaro, H., Lee, H. J., Hanson, L. G., Kim, H., Kuster, N., Siebner, H. R., Neufeld, E., \& Thielscher, A. (2020). Transducer modeling for accurate acoustic simulations of transcranial focused ultrasound stimulation. Journal of Neural Engineering, 17(4), [046010]. https://doi.org/10.1088/1741-2552/ab98dc

\section{General rights}

Copyright and moral rights for the publications made accessible in the public portal are retained by the authors and/or other copyright owners and it is a condition of accessing publications that users recognise and abide by the legal requirements associated with these rights.

- Users may download and print one copy of any publication from the public portal for the purpose of private study or research.

- You may not further distribute the material or use it for any profit-making activity or commercial gain

- You may freely distribute the URL identifying the publication in the public portal 


\title{
Transducer modeling for accurate acoustic simulations of transcranial focused ultrasound stimulation
}

\author{
Cristina Pasquinelli, ${ }^{\mathrm{a}, \mathrm{b}, \mathrm{h}}$, Hazael Montanaro ${ }^{\mathrm{c}, \mathrm{d}, \mathrm{h}}$, Hyunjoo J. Lee $^{\mathrm{e}}$ Lars G. Hanson $^{\mathrm{a}, \mathrm{b}}$, \\ Hyungguk Kim ${ }^{\mathrm{e}}$, Niels Kuster ${ }^{\mathrm{c}, \mathrm{d}}$, Hartwig R. Siebner ${ }^{\mathrm{a}, \mathrm{f}, \mathrm{g}}$, Esra Neufeld ${ }^{\mathrm{c}}$, Axel Thielscher ${ }^{\mathrm{a}, \mathrm{b}}$ \\ ${ }^{a}$ Danish Research Centre for Magnetic Resonance, Centre for Functional and Diagnostic Imaging and \\ Research, Copenhagen University Hospital Hvidovre, Denmark \\ ${ }^{b}$ Center for Magnetic Resonance, Department of Health Technology, Technical University of Denmark, \\ Kgs. Lyngby, Denmark \\ ${ }^{c}$ Foundation for Research on Information Technologies in Society (IT'IS), Zurich, Switzerland \\ ${ }^{d}$ Department of Information Technology and Electrical Engineering, Swiss Federal Institute of Technology \\ (ETH), Zurich, Switzerland \\ ${ }^{e}$ School of Electrical Engineering, Korea Advanced Institute of Science and Technology, Daejeon, South \\ Korea \\ ${ }^{f}$ Department of Neurology, Copenhagen University Hospital Bispebjerg, Copenhagen, Denmark \\ ${ }^{g}$ Institute of Clinical Medicine, Faculty of Health and Medical Sciences, University of Copenhagen, \\ Copenhagen, Denmark \\ ${ }^{h}$ The authors contributed equally to the work
}

\begin{abstract}
Objective: Low-intensity transcranial ultrasound stimulation (TUS) is emerging as noninvasive brain stimulation technique with superior spatial resolution and the ability to reach deep brain areas. Medical image-based computational modeling could be an important tool for individualized TUS dose control and targeting optimization, but requires further validation. This study aims to assess the impact of the transducer model on the accuracy of the simulations.
\end{abstract}

Approach: Using hydrophone measurements, the acoustic beam of a single-element focused transducer (SEFT) with a flat piezoelectric disc and an acoustic lens was characterized. The acoustic beam was assessed in a homogeneous water bath and after transmission through obstacles (3D-printed shapes and skull samples). The acoustic simulations employed the finite-difference time-domain method and were informed by computed tomography (CT) images of the obstacles. Transducer models of varying complexity were tested, representing the SEFT either as a surface boundary condition with variable curvature, or also accounting for its internal geometry. In addition, a back-propagated pressure distribution from the first measurement plane was used as source model. The simulations and measurements were quantitatively compared using key metrics for peak location, focus size, intensity and spatial distribution.

Main results: While a surface boundary with an adapted, 'effective' curvature radius based on the specifications given by the manufacturer could reproduce the measured focus location and size in a homogeneous water bath, it regularly failed to accurately predict the beam after obstacle transmission. In contrast, models that were based on a one-time 
calibration to the homogeneous water bath measurements performed substantially better in all cases with obstacles. For one of the 3D-printed obstacles, the simulated intensities deviated substantially from the measured ones, irrespective of the transducer model. We attribute this finding to a standing wave effect, and further studies should clarify its relevance for accurate simulations of skull transmission.

Significance: Validated transducer models are important to ensure accurate simulations of the acoustic beam of SEFTs, in particular in the presence of obstacles such as the skull.

Keywords:

transcranial focused ultrasound stimulation, single element focused transducer, finite difference time domain, computational dosimetry, Gamma method

\section{Introduction}

Transcranial focused ultrasound (TUS) has been successfully applied for stereotactic neurosurgery, brain tumor ablation, and reversible blood brain barrier (BBB) disruption. More recently, TUS has also emerged as a promising non-invasive brain stimulation technique due to the smaller focal size and the possibility to reach deeper brain areas compared to other non-invasive stimulation techniques [1]. Both excitatory and inhibitory neuromodulatory effects of TUS have been repeatedly demonstrated in several animal species, including nonhuman primates $[2,3,4,5,6]$ and humans $[7,8,9,10]$.

Safe application of TUS requires the precise control of the ultrasound dose in the brain. So far, this mostly relates to the control of the focus position and size of single element focused transducers (SEFT) that have been used in the majority of studies due to their relative ease of use and low cost, despite a more limited control of the energy deposition and spatial targeting compared to multi-element phased array transducers. The SEFT's beam profile can be characterized using hydrophone acoustic pressure measurements both in a homogeneous water environment and after the transmission through skull samples. These measurements, however, allow only for a limited assessment of the actual TUS dose in human in-vivo applications as the beam profile depends strongly on the skull's heterogeneous and individually varying structure and thickness [11]. Computer simulations informed by imaging techniques like magnetic resonance imaging (MRI) and computed tomography (CT) could play an important role in a non-invasive dose control and treatment planning on an individual patient basis. Simulations typically make use of some numerical method (e.g., the finite difference time domain - FDTD method) to solve the pressure wave equation [12] for modeling the propagation of acoustic waves through inhomogeneous media. Their usefulness for dose control in practical applications directly depends on their accuracy.

Much prior work has focused on the geometry and acoustic properties of the skull [13, $14,15,16,17,18]$, as inadequate skull models will cause significant errors in transcranial simulations. However, special care also must be taken when modeling the transducer device. SEFTs typically consist of either a physically curved vibrating piezoelectric element or a flat piezoelectric disk with an acoustic lens shaped like a concave spherical cap. For the second case, SEFTs are frequently modelled by representing only the forward facing, spherical-cap- 
shaped surface and imposing a pressure or velocity boundary condition on it, neglecting the internal structure of the device. The simulated beam profile of this simplified model differs from the real profile when modeling the transducer surface curvature according to its real curvature, as shown in Figure 3-B. Manufacturers therefore typically report an effective curvature to match the experimentally measured focus location [19] in a homogeneous water bath, as shown in Figure 3-C. Simulations then model the transducer by using this reported effective curvature or by adapting the simulated curvature to fit experimental data on the focus location, and ignore the real and internal transducer geometry [20, 11, 21, 22, 23].

While this approach allows matching the experimental location of the beam focus and its size (i.e., the peak dose location and full width at half maximum) in a test tank with water as medium and no obstacle, further validation is required when moving to more complex and heterogeneous obstacles, such as the skull. In this study, we used a SEFT with a flat piezoelectric disk and an acoustic lens and compared the simplified effective transducer model, as per the manufacturer's specification $\left(\mathrm{S}_{\text {eff }}\right)$, against (1) physically realistic and detailed models of the transducer accounting for its internal geometry $\left(\mathrm{P}_{1-2}\right.$; see Section 2.7), and (2) a source model derived by back-propagation $\left(\mathrm{M}_{\mathrm{B}-\mathrm{P}}\right)$ of the measured pressure distribution to a virtual source plane. We investigated the sensitivity of the new models to the different underlying parameters and assessed their accuracy by comparing the results with hydrophone measurements of the beam in the presence of different obstacles. Obstacles consisted of two bone samples (sheep and pig) and three 3D-printed simple and skull-shaped phantoms made of Veroblack, a material of known acoustic properties. In addition, we acquired CT data of the obstacles with the transducer and obstacle holder for precise positioning and to derive estimates of the obstacle's geometry and acoustic property distributions in the case of the bone skulls. Finally, we assessed the sensitivity of simulations to the modeling parameters when employing full human head models instead of the obstacles. Our results show that the simplified, effective transducer model that was based on the specification sheet of the manufacturer produces substantial deviations between the simulations and measurements. Specifically, clear deviations occurred in the presence of skull obstacles even though the correspondence was reasonable for the case of a homogeneous water background. This effect is even larger for a model that assigns a boundary condition to a surface reproducing the real, geometrically correct (physical curvature radius) transducer surface shape $\left(\mathrm{S}_{\text {geom }}\right)$. The deviations were substantially reduced for the transducer models that were established based on calibration measurements in a pure water background.

\section{Methods}

Figure 1 gives an overview of the methodological workflow, which is discussed in detail in the next sections.

\subsection{Bone samples and phantoms}

In this study, two animal skull samples (pig and sheep; see Figure 1-A) and three phantoms were tested. The pig skull was bought from a butcher and the sheep skull was donated by a local farmer. Soft tissue from both skulls was mechanically removed with tweezers, and 
A)

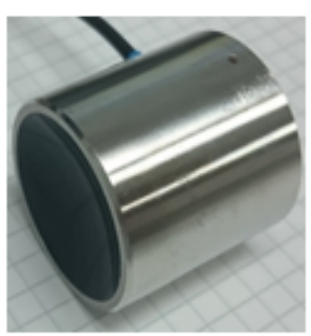

B)

B) CT data

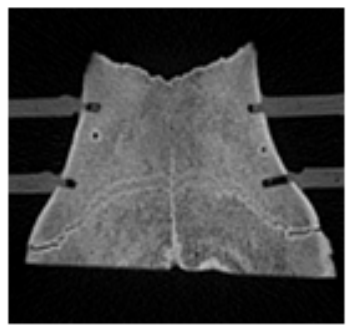

Transducer and obstacles
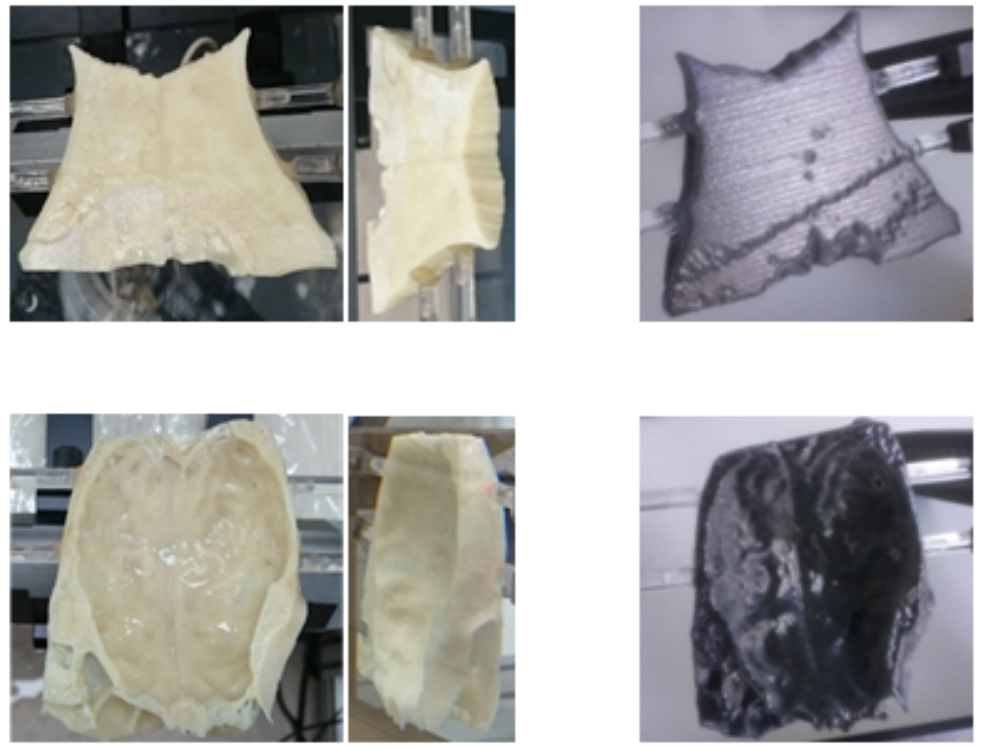

D) Simulations

C) Acoustic property

$$
\text { mapping }
$$

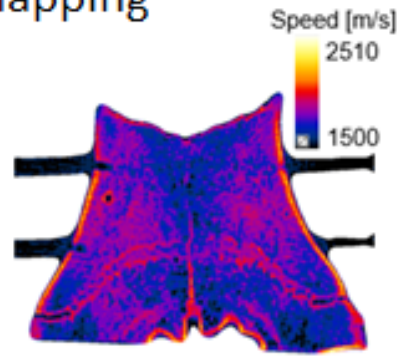

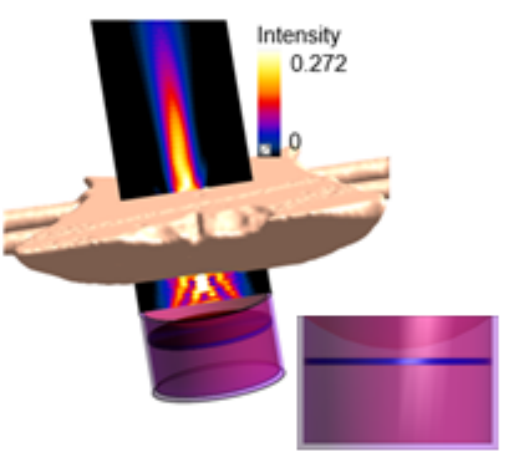

Figure 1: Workflow in the methods section. (A) The propagation of an ultrasound transducer (left) was characterized in a pure water background and after the transmission through obstacles. Specifically, the obstacles were a pig and a sheep skull (center, top and bottom, respectively), and the corresponding 3D printed phantoms (right). (B) The CT data of the obstacles were acquired, (C) to map their acoustic properties and to import their geometry in the simulation environment. (D) An example of the normalized intensity beam with the pig skull obstacle is shown, together with a view of the transducer model based on its actual internal geometry. 
the samples were cut to maintain the upper parts of the skull. While the entire upper part of the sheep skull was preserved, only part of the pig skull was extracted to have a thickness comparable with that of the human cranium. A mostly flat surface was obtained where the pig skull was cut. The two bone samples were then glued to holders (see Figure 2) and subsequently continuously kept under phosphate-buffered saline (PBS) solution. The skulls were not degassed, but the CT data acquired before and after the measurements did not reveal the presence of gas inside the samples. In addition, three 3D-printed phantoms were constructed from Veroblack (acoustic properties in Table 1). Two of those phantoms replicated the outer shape of the pig and sheep skull samples, as reconstructed from CT data (Figure 1-A). The third phantom consisted of a printed rectangular slab obstacle $\left(100 \times 100 \times 5 \mathrm{~mm}^{3}\right)$.

\begin{tabular}{lrrrr}
\hline Material & $\rho\left[\mathrm{kg} / \mathrm{m}^{3}\right]$ & $c[\mathrm{~m} / \mathrm{s}]$ & $\alpha[\mathrm{Np} / \mathrm{m}]$ & $Z[$ MRayl $]$ \\
\hline Water (generic) & 1000 & 1500 & 0 & 1.5 \\
Water $\left(20^{\circ} \mathrm{C}\right)$ & 1000 & 1482 & 0 & 1.5 \\
Veroblack & 1180 & 2495 & 21.3 & 2.9 \\
Acrylic Resin & 1190 & 2750 & 7.3 & 3.3 \\
Acrylic M1-7 & 1180 & 2610 & 14.2 & 3.1 \\
Human Cortical Skull & 1908 & 2814 & 27.2 & 5.4 \\
\hline
\end{tabular}

Table 1: Acoustic properties of different materials used in this paper, for a center frequency of $f=500 \mathrm{kHz}$ from $[24,11,25]$. In particular, the density $(\rho)$, the speed of sound $(c)$, the coefficient of attenuation $(\alpha)$ and the acoustic impedance $(Z)$ are indicated for each material. Different values for speed-of-sound in water that were used in the uncertainty assessment are included (see Section 2.6).

\subsection{US transducer and water tank measurements}

A single element spherical ultrasound transducer element with a flat piezoelectric disc and an acoustic lens (IPBD2, Hagisonic, South Korea, shown in Figure 1-A) operating at $500 \mathrm{kHz}$, with an aperture diameter of $3 \mathrm{~cm}$, a radius of curvature of $2.5 \mathrm{~cm}$, and a reported focal length of $5 \mathrm{~cm}$ (incorrectly reported as radius of curvature by the manufacturer specification sheet [19]), was used to generate the acoustic pressure waves. The setup used for the bone samples employed two function generators (33220A, Agilent Technologies, California, United States) to generate a burst (20 pulses / burst) of sinusoidal waves with a center frequency of $500 \mathrm{kHz}$ at a pulse repetition frequency of $1 \mathrm{kHz}$. The pulse was subsequently amplified by a power amplifier (5312, OPHIR, California, USA) and sent to the transducer. We first measured the pressure distribution along the symmetry axis from far to near field with different numbers of pulses (from 2 up to 30) per burst. In the far field, we observed that the measured beam intensity did not change beyond 15 pulses, meaning that the steady-state was reached. We chose a number of pulses per burst of 20, which, considering a speed of sound in water of $1500 \mathrm{~m} / \mathrm{s}$, corresponds to a travelled distance of $6 \mathrm{~cm}$. A custom-designed 3D-printed holder was used to fix the transducer inside a tank filled with de-ionized water. The pressure wave was sampled with a calibrated needle hydrophone with an active diameter of $1 \mathrm{~mm}$ (NH1000, Precision Acoustic, Dorset, UK) carefully inserted in a holder 
surrounded by a sponge in order to minimize reflection, as tested for continuous waves. The sponge is an open-cell foam for packing material (see Figure 2-A). More importantly, since the distance between the transducer and hydrophone holder was greater than $8 \mathrm{~cm}$ even when the hydrophone was close to the transducer, reflections from the holder did not affect the measurements of the 20 pulse bursts. Before each measurement series, the position of the transducer inside the holder was measured using a caliper. The hydrophone was moved by a stepper-motor system (Sciencetown Co., Incheon, South Korea) with a plane sampling distance of $0.25 \mathrm{~mm}$ and controlled by custom written software in Matlab. The signal from the hydrophone was transmitted and visualized with an oscilloscope (DSOX2022A, Agilent Technologies, California, United States). The raw data for each acquisition point were sent via USB to a computer and stored. In order to decrease noise, the signal was acquired using the average mode of the oscilloscope, with 32 samples averaged at each measurement position. For logistic reasons, the measurement setup used for the 3D-printed phantoms was changed to employ a different function generator $(33500 \mathrm{~B}$, Keysight, California, United States), amplifier (240L, E\&I, New York, United States) and oscilloscope (DSO-X 3024A, Agilent Technologies, United States), and an in-plane sampling resolution of $0.3 \mathrm{~mm}$. The material of the hydrophone holder was Plexiglas in these measurements, rather than aluminum, to improve the acoustic impedance matching with water. Measurements of the beam profile in water confirmed that this change did not affect the recorded data.

Thirteen planes parallel and one perpendicular to the transducer aperture were acquired to fully characterize the beam profile. The distance between twelve of the parallel planes was $5 \mathrm{~mm}$ and an additional plane near the focus was acquired at a distance of $2.25 \mathrm{~mm}$ from the nearest plane to better sample the strong spatial variations of the beam. The axial distance between the nearest parallel plane and the center of the transducer was $2 \mathrm{~cm}$. The apertureperpendicular plane was chosen to traverse the beam position of maximum intensity. In the case a strong secondary focus was detected, an additional perpendicular plane was acquired to cover it appropriately. The number of points for each acquired plane varied. The largest plane had a size of $3 \times 8 \mathrm{~cm}^{2}$ with a sampling distance of $0.25 \mathrm{~mm}$ resulting in 38841 points. The smaller planes had a size of $0.9 \times 0.9 \mathrm{~cm}^{2}$ with a sampling distance of $0.3 \mathrm{~mm}(961$ points in total). Prior to each plane measurement, the position of maximum intensity in water was determined as follows. First, two parallel planes with a separation of $2 \mathrm{~cm}$ were acquired near the focus and the positions of the peak intensities in each plane determined. Subsequently, measurements were performed along a line through these two positions. The obstacle was then put in place and several planes were acquired as stated above.

\subsection{Actual measurements with objects}

In order to precisely position the objects in the water tank, a holder was 3D-printed for each obstacle. This holder allows for the obstacle to be screwed in place at one of 5 different locations (position 1, 2A, 2B, 2C, and 3; see Figure 2).

The exact distance between transducer and obstacle was determined as follows: first, the location of the peak was determined in a water tank. Its position was recorded and used to define the symmetry axis. Then, the obstacle and its holder were inserted in the water tank. The distance along the symmetry axis between the forward facing obstacle surface and the 
previously determined focus location (in the absence of the obstacle) was measured. These measurements are used to accurately determine the position of the obstacle relative to the transducer. The complete set of obstacle distances to the transducer are shown in Figure 2. Beam profiles were measured after transmission through the two animal skull samples. A similar procedure was followed for the three 3D-printed Veroblack phantoms.

\subsection{Calculation of US intensity from the measured data}

The stored raw signals were first filtered with a high pass $4^{\text {th }}$ order Butterworth filter with a cutoff frequency of $200 \mathrm{kHz}$ to remove low-frequency noise. For each measured position, the intensity in $\mathrm{W} / \mathrm{m}^{2}$ was then calculated as

$$
I=\frac{p^{2}}{2 \rho c}
$$

where $p$ is pressure in $\mathrm{Pa}, \rho$ is the density of water $\left(1000 \mathrm{~kg} / \mathrm{m}^{3}\right)$ and $c$ is the speed-of-sound in water $(1500 \mathrm{~m} / \mathrm{s})$. To calculate the signal phase, a Hanning window was applied to the time domain signal to select only a central period (10th pulse). The Fourier transform was then calculated and the phase for the center frequency determined.

\subsection{CT imaging of the objects}

We acquired CT data of all objects attached to their holders both in water and air backgrounds using a PET / CT scanner (positron emission tomography, Biograph 128, Siemens, Germany). For each object, we acquired CT data with tube current-time product of $115 \mathrm{mAs}$ and tube potential of $80 \mathrm{kV}$, which would correspond to a low dose of $0.3 \mathrm{mSv}$ for a human head scan (roughly one third of the dose of clinical head scans). A sharp filter (H60s) was used during reconstruction. The nominal spatial resolution of the reconstructed images was $0.36 \times 0.36 \times 0.6 \mathrm{~mm}^{3}$. An example of one slice of the CT data for the pig skull is shown in Figure 1-B.

\subsection{Simulation framework}

Acoustic propagation was simulated within the Sim4Life (ZMT Zurich MedTech AG, Zurich, Switzerland) platform for computational life sciences, which encompasses functionality for image-based modeling, a range of acoustic propagation solvers, a Python scripting interface, as well as post-processing, visualization, and analysis functionality. For the purpose of this study, the linear acoustic pressure wave solver (LAPWE) from [12] was employed, which implements FDTD on rectilinear, inhomogeneous meshes and supports multiple graphical processing units (GPU) parallel execution to permit simulation of models with a large number of degrees-of-freedom within reasonable time. The LAPWE solver solves the wave equation:

$$
\begin{gathered}
\rho \nabla \frac{1}{\rho} \nabla p-\frac{1}{c^{2}} \frac{\partial^{2} p}{\partial t^{2}}-\frac{\tilde{a}}{c^{2}} \frac{\partial p}{\partial t}=0 \\
\tilde{a}=2 \alpha \sqrt{\frac{\alpha^{2} c^{4}}{\omega^{2}}+c^{2}} \\
7
\end{gathered}
$$




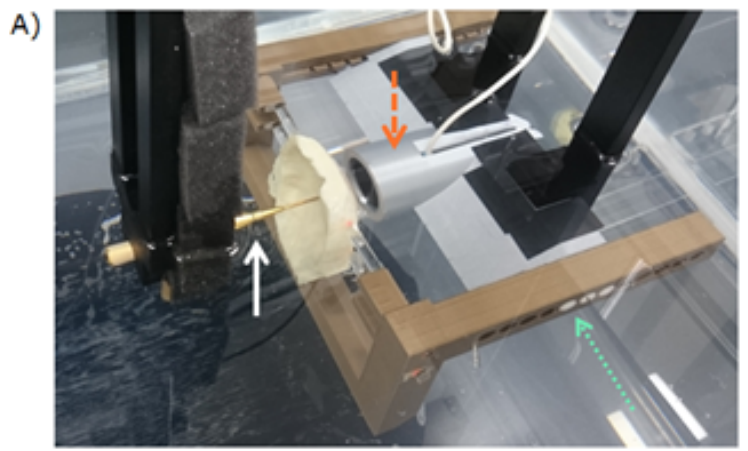

B)

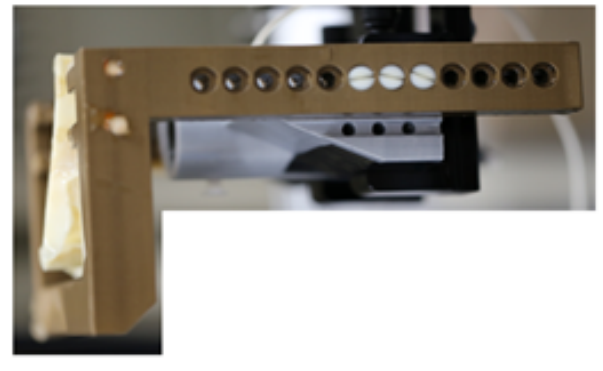

C)

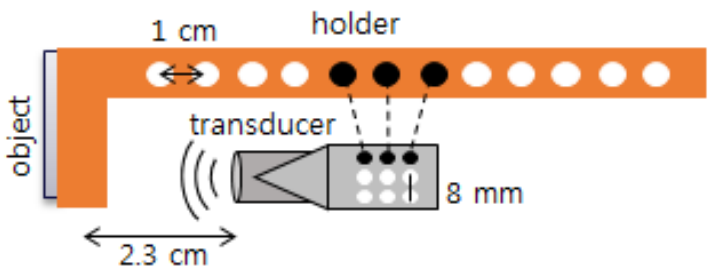

Position 1
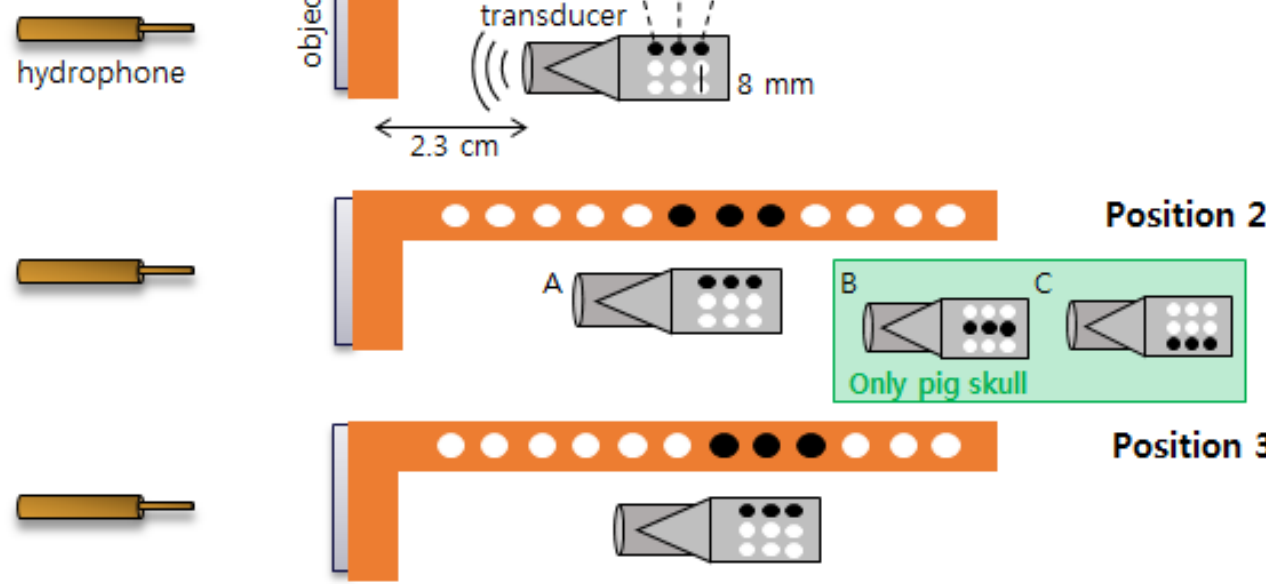

00000000000

Position 3
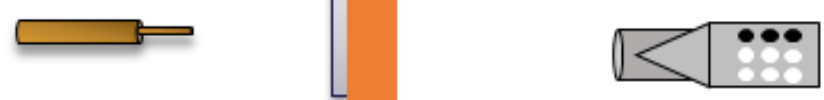

Figure 2: Details of the measurement setup. (A) The orange arrow points to the transducer, inside its 3D-printed holder (silver plastic). The transducer holder has an opening that allows the cable from the transducer to easily reach the amplifier and the curved face of the transducer to be positioned towards the hydrophone and the obstacles. The skull holder (gold plastic) is connected to the transducer holder with screws, in a way which is stylized by the black dotted line. The obstacle (here, the sheep skull) is glued to thin acrylic rods that are fixated in holes of the main part of the holder. The distance between the outer part of the transducer holder and the acrylic rods is $2.3 \mathrm{~cm}$, as indicated in the figure. The white arrow points to the hydrophone. It is fixed in a holder, which is surrounded by sponge to absorb acoustic reflections. While the transducer and the skull stay in the same position throughout a measurement, the hydrophone holder is moved by a stepper-motor system. (B) Holders of the hydrophone and object seen from the side (indicated by the green arrow in A). The relative position of the skull and the transducer can be changed in steps using different screw holes. (C) A schematic of the employed configurations in this study. Black dots indicate the position of the screws, and the transducer holder is shown in grey. The transducer face is inside the cylindrical part of the holder, as displayed in the figure. The horizontal distance of the holes in the skull holder is $1 \mathrm{~cm}$ and the vertical one is $8 \mathrm{~mm}$. 
where $\rho$ is density in $\mathrm{kg} / \mathrm{m}^{3}, c$ is speed-of-sound in $\mathrm{m} / \mathrm{s}, \alpha$ is attenuation in $\mathrm{Np} / \mathrm{m}, p$ is pressure in $\mathrm{Pa}, t$ is time in $\mathrm{s}$, and $\omega$ is angular frequency in $\mathrm{rad} / \mathrm{s}$. This equation accounts for reflections due to acoustic impedance $\left(Z=\rho c\right.$ in $\mathrm{kg} /\left(\mathrm{m}^{2} \mathrm{~s}\right)$ or Rayl) variations and discontinuities, standing waves, and the combined impact of absorption and scattering. However, it neglects shear waves in the rigid skulls, as well as tissue non-linearities, which can lead to frequency mixing and higher harmonics, but are not relevant at the studied intensities [13]. Grid generation ensured that the voxel size in every material remained below a tenth of its wavelength. The coarsest grid step was $0.3 \mathrm{~mm}$ outside the skull region and $0.1 \mathrm{~mm}$ in the skull region, resulting in a simulation mesh with about 500 million voxels. To ascertain the suitability of the discretization, a grid convergence analysis was performed for all obstacles in the holder's first position, where the grid resolution was increased until the peak amplitude change remained below 1\%. Perfectly Matched Layers (PML) boundary conditions with 16 layers were used to ascertain that reflections at the domain boundary remain negligible $(<2 \%$ amplitude change [26]). Undulations are still apparent in some of the simulations (see Figure 3). These result from imperfect wave absorption at the boundaries. It has been confirmed that enlarging the domain or increasing the number of PML layers indeed strongly reduces the undulations, at the cost of increased computation times. Detailed information about the verification and validation of this solver and its implementation can be found in [26].

In addition to performing simulations using the LAPWE solver, similar simulations were executed (for the sheep skull setup) with Sim4Life's Westervelt-Lighthill Equation (WLE) solver. This solver implements a higher-order (larger stencil) finite-difference scheme and also accounts for non-linearity (e.g., frequency mixing) and a first order frequency dependence of acoustic properties. These simulations allow to assess the impact of the numerical solver implementation, as well as the impact of neglecting non-linearity. The non-linearity parameter of $B / A=374$ was assigned according to [27], which is described as a conservative choice [28].

Simulations of the experimental setup were performed for a pure water background, as well as for the two skulls and the 3D-printed Veroblack plate and skulls, at up to five positions each (see Figure 2). The skull models were generated by thresholding the normalized CT images at 500 Hounsfield Units (HU) and extracting the skull component. Image upsampling (from $0.36 \mathrm{~mm}$ base resolution to $0.111 \mathrm{~mm}$ resolution) and Gaussian smoothing $(\sigma=1 \mathrm{~mm})$ were applied to avoid surface staircasing related to the CT resolution which is large compared to the discretization step in the simulation domain $(0.1 \mathrm{~mm})$. The transducer holder geometry was readily available from the computer-aided-design model used for rapid prototyping. For more details on the transducer geometry modeling, see Section 2.7. Reflections from the hydrophone and the tank walls were not simulated and had a very low impact on the measurement data because of the use of pulsed rather than continuous sonication. Using sufficiently short pulses prevents returning (reflected) waves from interfering with the outgoing wave in the measurement volume, due to the different arrival times.

Water was assigned generic acoustic properties (speed-of-sound: $1500 \mathrm{~m} / \mathrm{s}$, attenuation: $0 \mathrm{~Np} / \mathrm{m}$ ) and the properties of Veroblack were set according to [11]. Some simulations also used $1482 \mathrm{~m} / \mathrm{s}$ as the speed-of-sound in water in accordance with the IT'IS database (value 
at $20^{\circ} \mathrm{C}$ from [24]). For the acrylic acoustic lens material, properties were assigned within the range provided for acrylic resin and acrylic M1-7 in [25]. Acoustic properties of the different materials used in simulations are listed in Table 1. Transducer acoustic lens properties are discussed in Section 2.7 and the employed values for the final models $\mathrm{P}_{1-2}$ can be found in Table 3. Further lens variations can be found in Figures A.11 and A.12. To simulate the inhomogeneity of the bone skulls, the approach from [16] was used, which assumes that the CT HU can be linearly mapped into bone density, which in turn maps to speed-of-sound according to the following linear relations:

$$
\begin{gathered}
\rho=\rho_{r e f_{1}}+\frac{\rho_{r e f_{2}}-\rho_{r e f_{1}}}{H U_{r e f_{2}}-H U_{r e f_{1}}} H U \\
c=c_{r e f_{1}}+\frac{c_{r e f_{2}}-c_{r e f_{1}}}{\rho_{r e f_{2}}-\rho_{r e f_{1}}} \rho
\end{gathered}
$$

where $r e f_{1}$ and $r e f_{2}$ refer to the reference values used to anchor the linear mappings. $r e f_{1}$ was chosen as water, and $r e f_{2}$ was set to species specific average skull properties (density and speed-of-sound). For simplicity, attenuation in the skull was set to a constant value that was experimentally adjusted to match the maximum measured intensity when the skull was placed in the holder's first position. Typically, acoustic skull attenuation is experimentally inflated and adjusted to account for microscopic backscattering effects that cannot be effectively captured by even high resolution CT images [11, 16, 29]. As discussed in Section 4, attenuation primarily impacts the overall field scaling, but has little impact on focus shape and position. Skull mapping parameters are provided in Table 2. In order to determine them, a histogram of the skull CT HU data was extracted and clipped at the HU of water $(0 \mathrm{HU})$. The very apparent subsequent peak towards higher HU was assumed to be the average HU in skull and assigned to the corresponding (species-specific) value, as found in the literature ([30] for pig, [22] for sheep).

HU-based property assignment was restricted to the skull region and linear interpolation was used to relate the finer voxel resolution $(0.1 \mathrm{~mm})$ to the coarser CT $(0.335 \mathrm{~mm})$. To efficiently handle inhomogeneity, voxels were assigned to 20 different bone classes based on HU binning. This permits precomputation and storage of update coefficient look-up tables for the GPU accelerated solver, thus increasing solver efficiency. An increase of the number of binning classes beyond 20 was found to not significantly affect the acoustic distributions anymore $(<1 \%$ change in peak intensity). Examples of a map of speed-of-sound in a cross section of pig skull and how the simulated beam appears are shown in Figure 1-C and D, respectively. Acquiring the CT data of the skulls allowed also to replicate the exact position of the skulls in the simulation, and to correctly model their thickness. The thickness of the skulls varied from 13 to $3.5 \mathrm{~mm}$ for the pig and from 6 to $8 \mathrm{~mm}$ for the sheep. The thickness of the sheep skulls along the ultrasound beam was $6 \mathrm{~mm}$, while for the pig skull it varied because we shifted vertically the skull (see Figure 2). The impact of geometry segmentation, skull thickness, and inhomogeneous mappings using different CT scanner parameters (including the one in this work) is analyzed in [31]. 


\begin{tabular}{lrrrrrrrrr}
\hline Skull & \multicolumn{3}{c}{$r e f_{1}$ (Water) } & & \multicolumn{3}{c}{$r e f_{2}$ (Average Skull) } & \\
\cline { 2 - 3 } & $\mathrm{HU}$ & $\rho\left[\mathrm{kg} / \mathrm{m}^{3}\right]$ & $c[\mathrm{~m} / \mathrm{s}]$ & & $\mathrm{HU}$ & $\rho\left[\mathrm{kg} / \mathrm{m}^{3}\right]$ & $c[\mathrm{~m} / \mathrm{s}]$ & $\alpha[\mathrm{Np} / \mathrm{m}]$ \\
\hline Pig & 0 & 1000 & 1500 & & 630 & 1260 & 1903 & 62.5 \\
Sheep & 0 & 1000 & 1500 & & 1400 & 1710 & 2500 & 100 \\
Human & 0 & 1000 & 1500 & & peak & 1908 & 2814 & 27 \\
\hline
\end{tabular}

Table 2: Parameters used for skull property mappings, based on the approach from [16], but using species specific values from [30] [22] and [24]. peak corresponds to the peak HU value of the histogram of the segmented human skull.

\subsection{Transducer modeling}

Acoustic sources were modeled as time-harmonic sinusoidally varying Dirichlet pressure boundary conditions. The transducer casing was treated as a perfect reflector (zero pressure). Initially, an effective transducer model (termed $S_{\text {eff }}$ in the following) was created using a spherical cap as pressure boundary, with an aperture diameter of $30 \mathrm{~mm}$ and an outer surface curvature radius of $50 \mathrm{~mm}$, as provided in the manufacturer specification sheet. To confirm the given specifications of this model, ten simulations with varying curvature radii $(25-70 \mathrm{~mm})$ were performed, while the aperture diameter was held constant $(30 \mathrm{~mm})$. It was found that a curvature radius of $50 \mathrm{~mm}$ indeed optimally reproduces the measurements in water, both in term of peak location and Gamma metric (see Section 2.8), as shown in Figure A.10.

As the curvature of the above effective model differed clearly from that of the real transducer surface, we explored the beam shape predicated by a model $\left(\mathrm{S}_{\text {geom }}\right)$ consisting of a simplified spherical cap as pressure boundary, but having the geometrical surface curvature (radius of $25 \mathrm{~mm}$ ). As shown in Figure 3-B and 4, $\mathrm{S}_{\text {geom }}$ expectedly failed to reproduce the intensity distribution in water, and was therefore excluded from further analyses.

A subsequent inquiry with the manufacturer revealed that the actual piezo element is in fact a flat piston and that an additional acrylic element with the measured surface shape is inserted on top of this disk (Figure 3). Hence, a detailed transducer model was constructed that features a flat disk, which is assigned a time-harmonic sinusoidally varying pressure distribution, while the curved acrylic resin element on top (acoustic lens) is treated as a passive medium that shapes the wave-front (Figure 1). The acoustic properties (density, speed-of-sound, and attenuation) of the acrylic layer were based on [25] and varied within the associated uncertainty range (see Figures A.11 and A.12) until simulation results matched the measured field in the obstacle-less water bath setup (using the Gamma metric introduced in Section 2.8 as criterion). The depth of the disk was set to a quarter of the wavelength in the lens as reported in [32] and also varied. Furthermore, a radial dependence $p(r)$ of the pressure $p_{0}$ on the disk was introduced (constant: $p(r)=p_{0}$, linear: $p(r)=p_{0}(1-r)$, cosine: $p(r)=$ $p_{0} \cos \left(\frac{\pi}{2} r\right)$, and spherical: $p(r)=p_{0} \sqrt{1-r^{2}}$ for $\left.r \in[0,1]\right)$ to reflect the potential impact of the transducer walls on the vibrational mode ('aperture function') of the piezo source. That way, two candidate physical transducer models $\left(\mathrm{P}_{1-2}\right.$; see Table 3$)$ were generated. These fitted 'physical' models and the 'effective' model were then used to analyze how well 
they are able to predict transcranial ultrasound intensity distributions. The LAPWE solver typically employs 'hard' boundary conditions (i.e., the pressure at the source is imposed as a time varying Dirichlet boundary condition). This results in reflections of incoming (e.g., back-scattered) waves, which might not be completely accurate. Furthermore, a hard source impacts the pressure wave from the physical transducer models even in the absence of an obstacle, due to internal reflection in the transducer (e.g., by the transducer casing). Hence, a special version of the solver implementing the opposite extreme ('soft' sources, i.e., continuous addition of pressure at the source location) was used to study the resulting exposure change.

Table 3 summarizes the main transducer models employed in this paper.

\begin{tabular}{l|c|c}
\hline Model & Description & Parameters \\
\hline \hline $\mathrm{S}_{\text {geom }}$ & $\begin{array}{c}\text { Curved element with the actual } \\
\text { transducer curvature }(25 \mathrm{~mm})\end{array}$ & - \\
\hline $\mathrm{S}_{\text {eff }}$ & $\begin{array}{c}\text { Curved element with a curvature optimized } \\
\text { to reproduce the focus location }(50 \mathrm{~mm})\end{array}$ & - \\
\hline $\mathrm{M}_{\mathrm{B}-\mathrm{P}}$ & Flat source, derived from holographic reconstruction & - \\
\hline $\mathrm{P}_{1}$ & Transducer with an internal structure, & $c=2600 \mathrm{~m} / \mathrm{s}$ \\
\hline $\mathrm{P}_{2}$ & flat element and cosine aperture function & $\alpha=50 \mathrm{~Np} / \mathrm{m}$ \\
\hline
\end{tabular}

Table 3: Main transducer models employed in this paper. Parameters $c$ and $\alpha$ are the speed of sound and attenuation properties, respectively, of the acrylic acoustic lens. $\mathrm{P}_{1}$ and $\mathrm{P}_{2}$ are the best physical candidate models. For them, the density of the acrylic acoustic lens was set to $\rho=1180 \mathrm{~kg} / \mathrm{m}^{3}$ and the distance of the piezo element (in wavelengths of the lens material $\lambda$ ) from the base of the lens surface was set to $d=0.25 \lambda$

\subsection{Metrics}

All simulated pressure distributions were normalized by the peak simulated pressure in the absence of a bone or printed skull obstacle. The following metrics were used for quantification and comparison purposes: (i) $d_{z}$ and $d$ : peak location as a measure of focus position (position along $z$-axis to quantify focal depth and $(x, y, z)$-position to quantify absolute focal shift); (ii) $\mathrm{I}_{\text {peak }}$ : peak intensity (normalized by the peak intensity in the absence of any obstacle); (iii) $\mathrm{FWHM}_{z}$ : extent of the focus full width at half maximum along the principal propagation axis (where available); (iv) $\mathrm{HWHM}_{z+}$ : half maximum past the location of the focus (or the length until the intensity has decayed by a factor of two, in case the obstacle prevents measuring the peak location); (v) Gamma: Gamma comparison value. These quantities were all obtained based on the field distribution past the obstacle. The metrics were selected (and adapted, as described above) depending on availability (particularly for configurations where obstacles are placed far from the transducer).

The Gamma comparison method was proposed by [33] to compare planned and administered radiological dose distributions. It permits to quantitatively compare fields that include 


\section{Transducer Models}
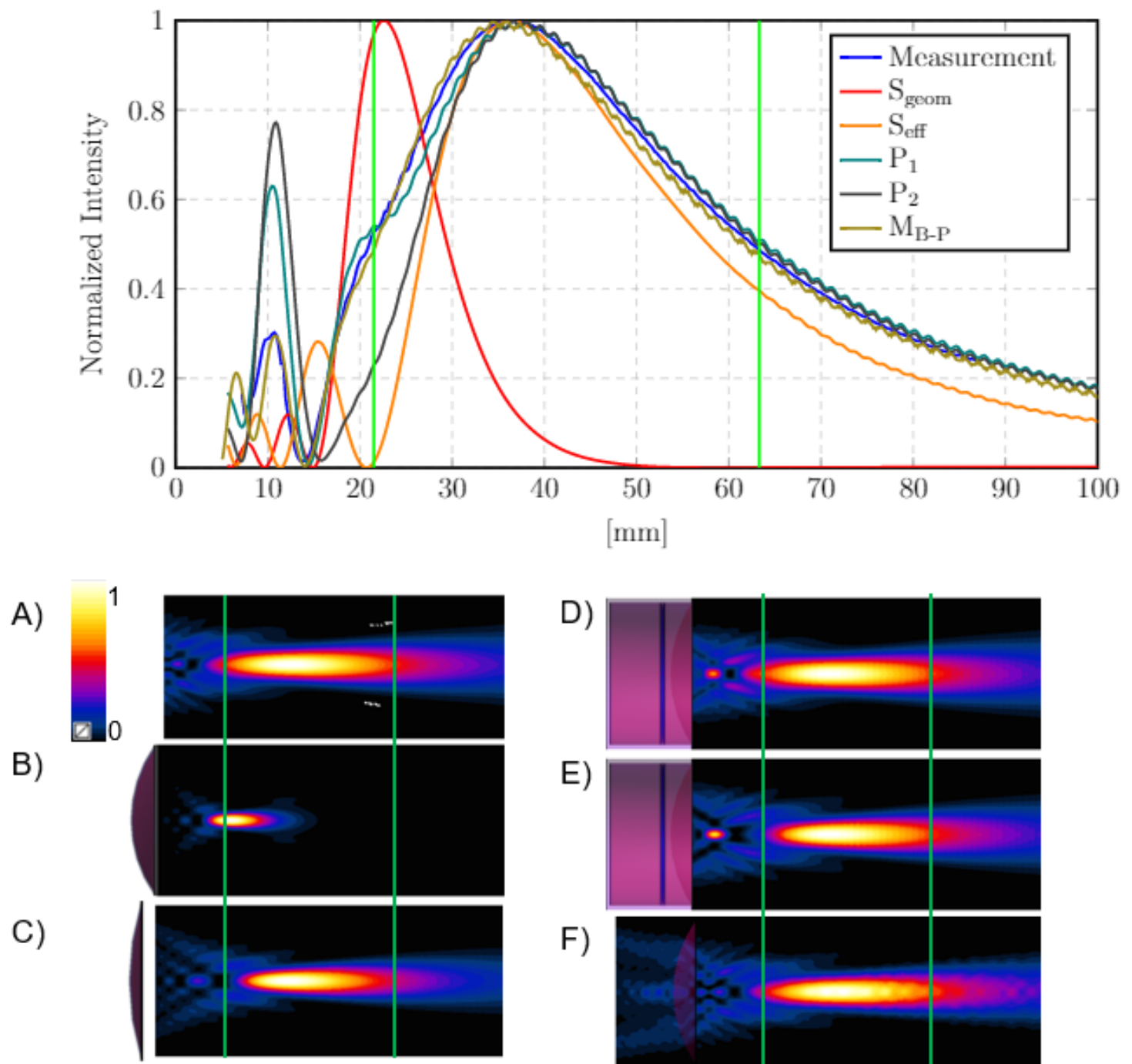

D)

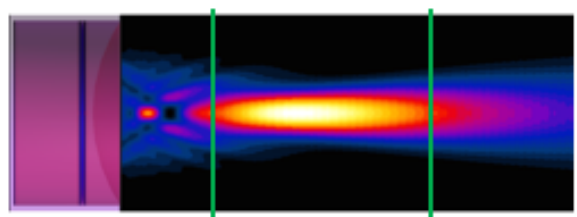

E)

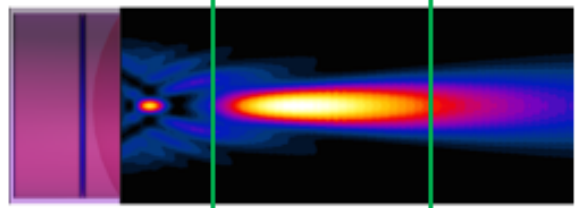

F)

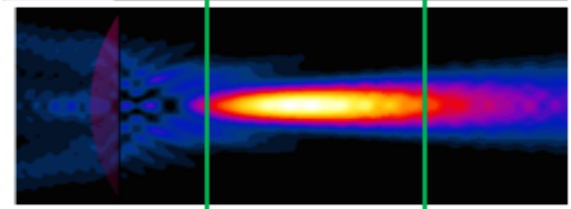

$\overleftarrow{10 \mathrm{~mm}}$

G)

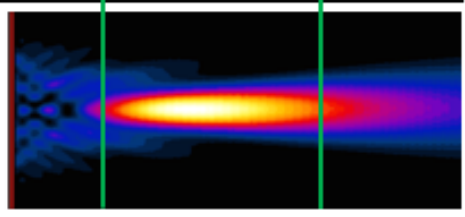

Figure 3: Transducer Models: (Top) Normalized acoustic intensity along the symmetry axis for different transducer models, compared to measurement data. The Full Width at Half Maximum extent of the measured focus is indicated by vertical green line. (A-G) Measured and simulated normalized intensity distributions. (A) Measurement, (B) $\mathrm{S}_{\text {geom }}$, (C) $\mathrm{S}_{\text {eff }}$, (D-E) $\mathrm{P}_{1-2}$ physical transducer models, (F) reconstruction using the plane-wave decomposition approach (from the first measurement plane), $(\mathrm{G})$ simulation using the back-propagated pressure from the first measurement plane to the transducer aperture plane as pressure source. 
both shape distortions and variations in amplitude, and its use in validation experiments has been advocated by [26]. Given spatial tolerances and an amplitude tolerance $\left(\Delta d_{x, y, z}\right.$ and $\Delta D$, respectively), the gamma index $(\gamma)$ compares every measurement point with all simulation points and finds the simulation point that minimizes an Euclidean distance norm combining distance and value $(f(\vec{r}))$ deviations (normalized by the corresponding tolerances):

$$
\Gamma\left(\vec{r}_{\text {model }}, \vec{r}_{\text {meas }, i}\right)=\sqrt{\frac{\left(f_{\text {model }}\left(\vec{r}_{\text {model }}\right)-f_{\text {meas }}\left(\vec{r}_{\text {meas }, i}\right)\right)^{2}}{\Delta D^{2}}+\sum_{j=x, y, z} \frac{\left(r_{j, \text { model }}-r_{j, \text { meas }, i}\right)^{2}}{\Delta d_{j}^{2}}}
$$

The minimized Euclidean distance norm is subsequently assigned as the score of the corresponding measurement location:

$$
\gamma\left(\vec{r}_{\text {meas }, i}\right)=\min \left\{\Gamma\left(\vec{r}_{\text {model }}, \vec{r}_{\text {meas }, i}\right)\right\} \forall \vec{r}_{\text {model }}
$$

A value of 0 corresponds to a perfect match for this point and a value of 1 reflects the limit of what lies within the total tolerance. The total tolerance is obtained as root-sumsquare reflecting the simplified assumption of statistical independence. We report the gamma comparison value 'Gamma' as the percentage of measurement points that have been assigned a norm exceeding 1 (outside of the tolerance). Furthermore, the spatial distribution of $\gamma\left(\vec{r}_{\text {meas }}\right)$ provides an intuitive visualization of disagreement locations (see Figure 4 for an illustrative example of the Gamma comparison method).

$$
\gamma\left(\vec{r}_{\text {meas }, i}\right)>1: \text { disagreement exceeds combined tolerance }
$$

The chosen agreement criteria were motivated by the intended application - i.e., the spatially precise targeting of a small cortical patch - and the physical beam properties. Given a focus size with a FWHM of $42 \mathrm{~mm}$ along the beam axis and $5 \mathrm{~mm}$ perpendicular to it (determined for pure water, see Figure 3), we set $\Delta d_{z}=5 \mathrm{~mm}$ and $\Delta d_{x y}=2 \mathrm{~mm}$ as upper thresholds for shifts of the focus position in these directions. Shifts exceeding these criteria would result in the undesired stimulation of a neighboring cortical patch, or in a peak position that is in cerebral spinal fluid (CSF) or white matter, rather than in gray matter. Based on the approximately sigmoidal dependence of TUS intensity on neural response as demonstrated in [34], a $15 \%$ difference in peak intensity maximally changes the stimulation success rate by $10 \%$. For this reason, $\Delta D=15 \%$ was set as the amplitude tolerance.

The same tolerance values were also used to normalize deviations in focus location and size. As we're measuring a euclidean distance for $d$, we used a single $5 \mathrm{~mm}$ deviation tolerance, independent of particular axial directions, for simplicity.

\subsection{Backpropagation}

To assess the choice of aperture functions (see Section 2.7), back-propagation from a plane parallel to the transducer aperture at an axial distance of $7.2 \mathrm{~mm}$ from the center of the transducer was performed (in the obstacle-free setup) and the resulting pressure distribution 


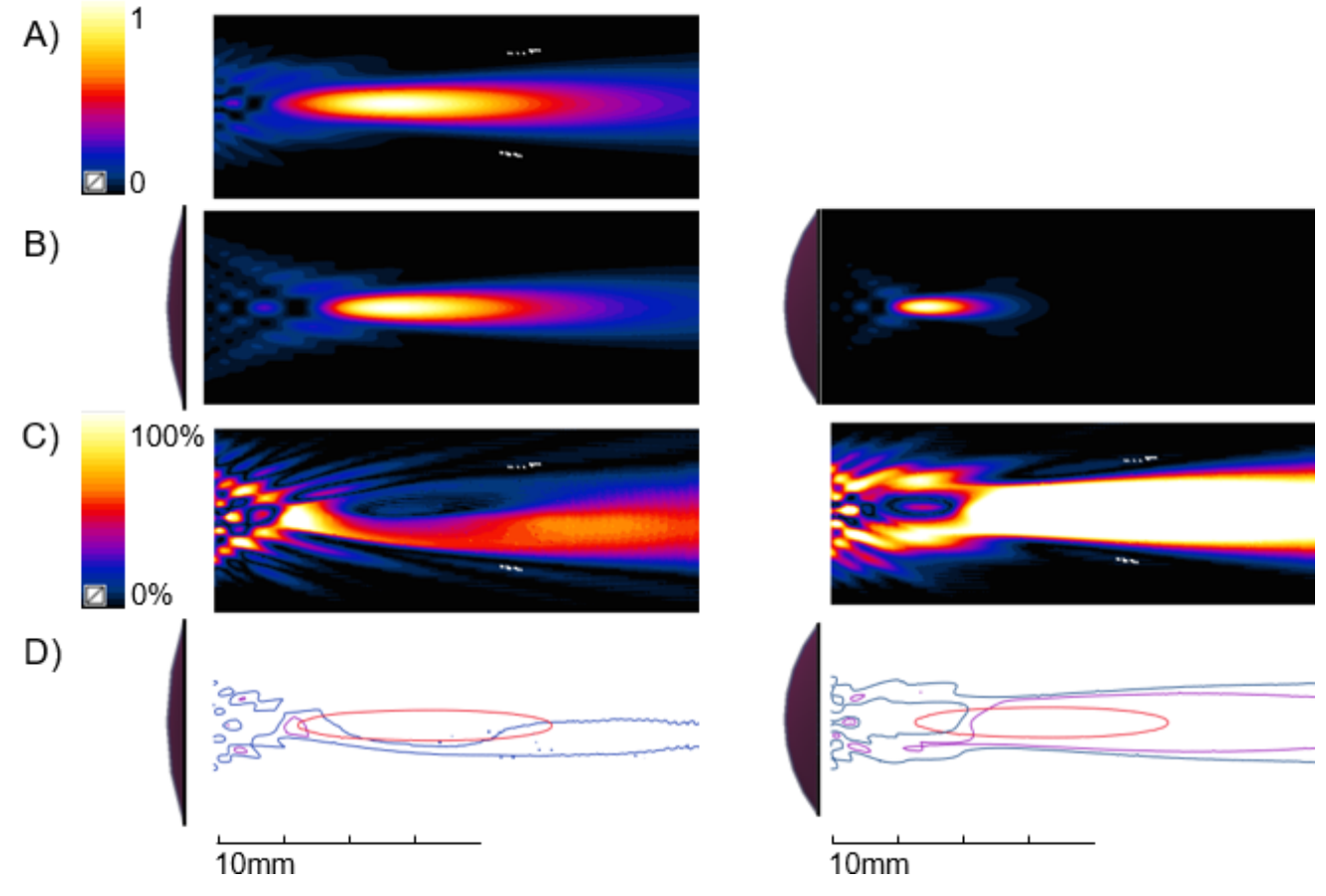

Figure 4: Illustrative example of a good (right) and a bad (left) Gamma comparison. (A) Measurement normalized to the peak intensity; (B) $\mathrm{S}_{\text {eff }}$, $\mathrm{S}_{\text {geom }}$; (C) Gamma comparison distribution $(\gamma(r)$; tolerances: $5 \mathrm{~mm}$ longitudinal, $2 \mathrm{~mm}$ transverse, and $15 \%$ intensity); (D) FWHM of measurement profile in red, isocurves of $\gamma(r)$ at $100 \%$ in purple and at $50 \%$ in blue. Notice that the biggest differences for $\mathrm{S}_{\text {eff }}$ occur in the near field and in the region of the focus - the latter indicating that the measurement focus does not align perfectly with the symmetry axis. A $\gamma$ value below $100 \%$ indicates deviation within the combined tolerance. The purple iso-curve demarks the regions that exceed the combined tolerance. For $\mathrm{S}_{\text {geom }}$, almost the entire region of the measurement focus is outside the acceptable tolerance. 
on the transducer surface was compared to those obtained in the different simulations. Backpropagation was performed using the plane-wave decomposition method (PWD, also known as angular spectrum method, $[35,36])$ in free space - neglecting the attenuation in water -, which precluded propagation beyond the transducer surface into the heterogeneous transducer structure. Evanescent modi were not exponentially increased in the back-propagation to avoid inflating measurement errors and sampling-related errors. Apodization and zeropadding were used before the Fourier transformation step of the plane-wave decomposition to reduce ringing and folding artifacts. The backpropagated pressure field on the plane encompassing the transducer aperture was used as source $\left(\mathrm{M}_{\mathrm{B}-\mathrm{P}}\right)$ in simulations with and without sheep skull obstacle.

\subsection{Human head models}

Simulations involving three different human head models (S1, S2, S3) from [37] were performed using the $\mathrm{P}_{1}$ and the $\mathrm{S}_{\text {eff }}$ transducer model (see Figure 8). These head models were created from multi-modal image data (different MRI sequences, as well as CT), permitting the consideration of skull heterogeneity. For a list of the 15 segmented tissues, see [37]. Tissue properties have been assigned according to [24], while the skull density and speed-of-sound were linearly mapped from the CT's HU according to Table 2 . While no reference measurements exist to assess the validity of the simulated pressure distribution, these simulations can serve to illustrate the principal impact of transducer modelling on simulated transcranial sonication.

\section{Results}

\subsection{Effects of transducer modeling on the acoustic beam in a pure water background}

Modeling the transducer as a pressure Dirichlet boundary condition on an 'effective' model that adapts the curvature radius in accordance with the effective radius provided by the manufacturer $\left(\mathrm{S}_{\text {eff }}\right)$ results in a low Gamma error $(0.2 \%$; Table 4$)$. We confirmed that the chosen effective radius best reproduced the focus position in water and minimized the Gamma metric in water (Figure A.10). The physics-based transducer models further improve the agreement (Gamma $=0 \%$ ), yet this is expected as they have been tuned to match water measurements. The impact of the various transducer parameters on the intensity distribution and along the symmetry axis is depicted in Figures A.11 and A.12.

When a 'soft' source is used instead of Dirichlet boundary conditions, transducer-internal reflections by structures behind the piezo-element become relevant. Due to the lack of knowledge about the internal transducer structure, the region within the casing and behind the piezo was modeled as homogeneous material with varying absorption (50 and $150 \mathrm{~Np} / \mathrm{m})$, while the casing was treated as perfect reflector. In comparison to the Dirichlet pressure boundary condition, the secondary foci in the near field are less prominent and the focus length is shortened by $37 \%$ (see Figure A.13). 


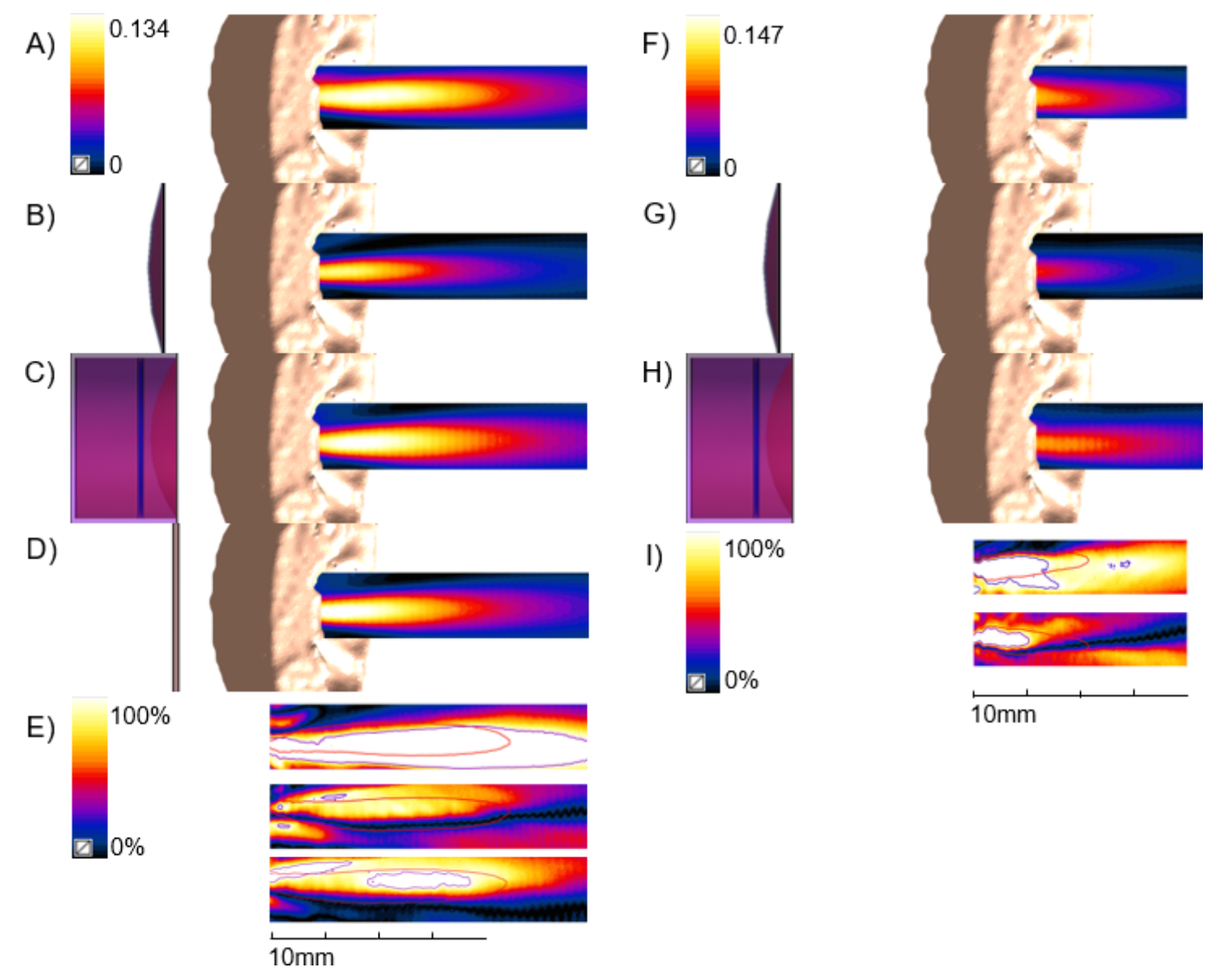

Figure 5: Sample measurement and simulation intensity distributions normalized to the peak intensity in water, and gamma comparison distribution past obstacles. (A-D) Illustrative 'good' simulation result (sheep skull, position 1) and (E-H) 'bad' simulation match (sheep skull, position 3) with measurement. $(\mathrm{A}, \mathrm{F})$ Measurement, (B,G) $\mathrm{S}_{\mathrm{eff}},(\mathrm{C}, \mathrm{H}) \mathrm{P}_{1}$ physics-based transducer model, (D) $\mathrm{M}_{\mathrm{B}-\mathrm{P}}$ plane source, (E,I) Gamma comparison of normalized deviation between measurement and different transducer models (from top to bottom, $\mathrm{S}_{\mathrm{eff}}, \mathrm{P}_{1}, \mathrm{M}_{\mathrm{B}-\mathrm{P}}$, where available). The overlaid red contour denotes the Half Maximum iso-contour of the measurement distribution; the purple contour indicates the region with a deviation that exceeds the combined tolerance. 


\begin{tabular}{lrrrrr}
\hline \multicolumn{6}{c}{ Watertank Comparison [\%] } \\
\hline & Gamma & \multicolumn{1}{c}{$d_{z}$} & \multicolumn{1}{c}{$d$} & $\mathrm{FWHM}_{z}$ & $\mathrm{HWHM}_{z+}$ \\
\hline $\mathrm{S}_{\text {eff }}$ & 0.2 & -26.5 & 28.7 & -166.1 & -90 \\
$\mathrm{P}_{1}$ & 0.0 & -13.3 & 18 & 33.6 & 28.7 \\
$\mathrm{P}_{2}$ & 0.0 & -16.3 & 19.8 & -55.4 & 27.7 \\
$\mathrm{M}_{\text {B-P }}$ & 0.0 & -16.3 & 22.4 & -25.7 & -0.01 \\
\hline
\end{tabular}

Table 4: Differences between the measured and simulated intensities in a watertank setup without obstacle. The differences are expressed in percentages normalized to the tolerances from Section 2.8.

\subsection{Transmission through the Veroblack plate}

Once the Veroblack plate obstacle is introduced, the 'effective' transducer model fails to reproduce focus location and size. The two physics-based transducer models, however, are capable of predicting the focus location, size, and overall pressure distribution reliably (0\% disagreement of the Gamma metric vs. $15 \%$ and $19 \%$ of the points failing the Gamma comparison for the effective transducer model at two different positions; see Table A.5).

\subsection{Transmission through the Veroblack 3D-printed skulls}

Pig (thick skull). The 3D-printed pig skull is the thickest Veroblack obstacle (the thickness along the propagation axis can exceed $10 \mathrm{~mm}$ ), resulting in standing wave patterns inside the obstacle. While one side is mostly flat (due to the cutting), the other is slanted such that small shifts in predicted focus position can result in clear changes of the standing waves in the obstacle and the related transmission efficacy (see Section 4). Depending on the skull position perpendicular to the propagation direction (positions $2 \mathrm{~A}-\mathrm{C}$ ), the beam goes through the maximal thickness or partly passes outside the skull fragment border $(2 \mathrm{C})$. This results in two separate, prominent focal lobes and some weak secondary foci. In all the Veroblack printed pig skull cases, the peak intensity is within the (unmeasurable) region inside the skull or near its surface. Therefore, while the Gamma comparison is meaningful, reported peak intensity $\left(\mathrm{I}_{\text {peak }}\right)$ and focus size comparisons $\left(\mathrm{HWHM}_{z+}\right)$ are not. The 'effective' model is unable to predict focus intensity (the error is smaller than the tolerance only for position 3) and frequently fails to correctly predict focus extent. Consequently, it displays poor Gamma metrics. The physics-based transducer models produce good results, except for positions $2 \mathrm{~B}$ and 3, where the intensity is off, despite very good agreement in the relative distribution pattern. The physics-based simulations are all well able to handle the challenging $2 \mathrm{C}$ case, where the focus is right at the border of the skull fragment (see Table A.6).

Sheep. The 3D-printed sheep skull is thinner and curved and the agreement between simulations and hydrophone intensity measurements is better than for the 3D-printed pig skull. The physics-based models outperform the 'effective' model, based on the Gamma criterion, and, with the exception of position 3 where the 'effective' model shows a $>30 \%$ Gamma error rate, the Gamma criterion remains below $5 \%$ for all transducer models. However, the focus position error frequently exceeds the chosen $5 \mathrm{~mm}$ tolerance (see Table A.7). 


\subsection{Transmission through the bone skull samples}

Pig. The physics-based transducer models show near perfect Gamma metrics (always $<1 \%$ ), whereas the percentage of data-points failing to agree with the hydrophone measurements (according to the Gamma criterion) can reach over $20 \%$ for the 'effective' transducer model. While the physics-based transducer model occasionally slightly exceeds the prescribed peak intensity tolerance of $15 \%$, the 'effective' model nearly always fails and exceeds the tolerance threshold by up to 4.8 times (see Table A.8).

Sheep. Simulation performance with the sheep skull obstacle is inferior to the accuracy achieved for the pig skull. However, the physics-based models again outperform the 'effective' model. The latter fails to pass the Gamma criterion for 7-40\% of the measurement points, while the failure rate of the former is in the 4-20\% range (see Figure 5). In most cases, the prediction of the peak intensity is insufficient (considering the defined $15 \%$ threshold), which seems to be related to the simulations predicting two distinct but overlapping intensity peaks, while the measurements show a single, merged peak of combined (higher) intensity (see Table A.8).

Combined Results. Figures 6 show the averaged deviation between the measured and simulated distributions for the different transducer models. The physical transducer models clearly outperform the 'effective' model in terms of the Gamma metric and the accuracy of the focus location and size predictions. However, it is also apparent, that none of the models can always guarantee predictions within the desired tolerances. This is particularly true with regard to the peak intensity prediction and in cases where standing-wave effects occur (e.g., Veroblack pig skull). The performance of the two physical transducer models is comparable.

\subsection{Other Parameters}

Water speed-of-sound. The speed-of-sound of water is temperature dependent. A slight difference in speed-of-sound in combination with a large distance (in terms of wavelengths) can result in a noticeable change of the interference pattern and pressure distribution. Changing $c_{\text {water }}$ from 1500 to $1482 \mathrm{~m} / \mathrm{s}$ has no visible impact on the pressure distribution in water. The impact is more pronounced after transmission through the sheep skull (see Table A.8). Particularly the peak position shifts significantly. However, inspection of the pressure distribution as well as the Gamma metric reveal that this is due to small changes in the region of a flat peak and that the distribution difference remains small.

\subsection{Backpropagation}

The backpropagated pressure distribution on the transducer surface is depicted in Figure 7 , which also shows the sonication intensity in water, as reconstructed from the first measurement plane using the angular spectrum method. The measured and reconstructed intensity distributions are in good agreement. When comparing the backpropagated radial dependence of the pressure with those obtained using the physical transducer models with varying aperture functions, it is found that the backpropagated one lies between the 

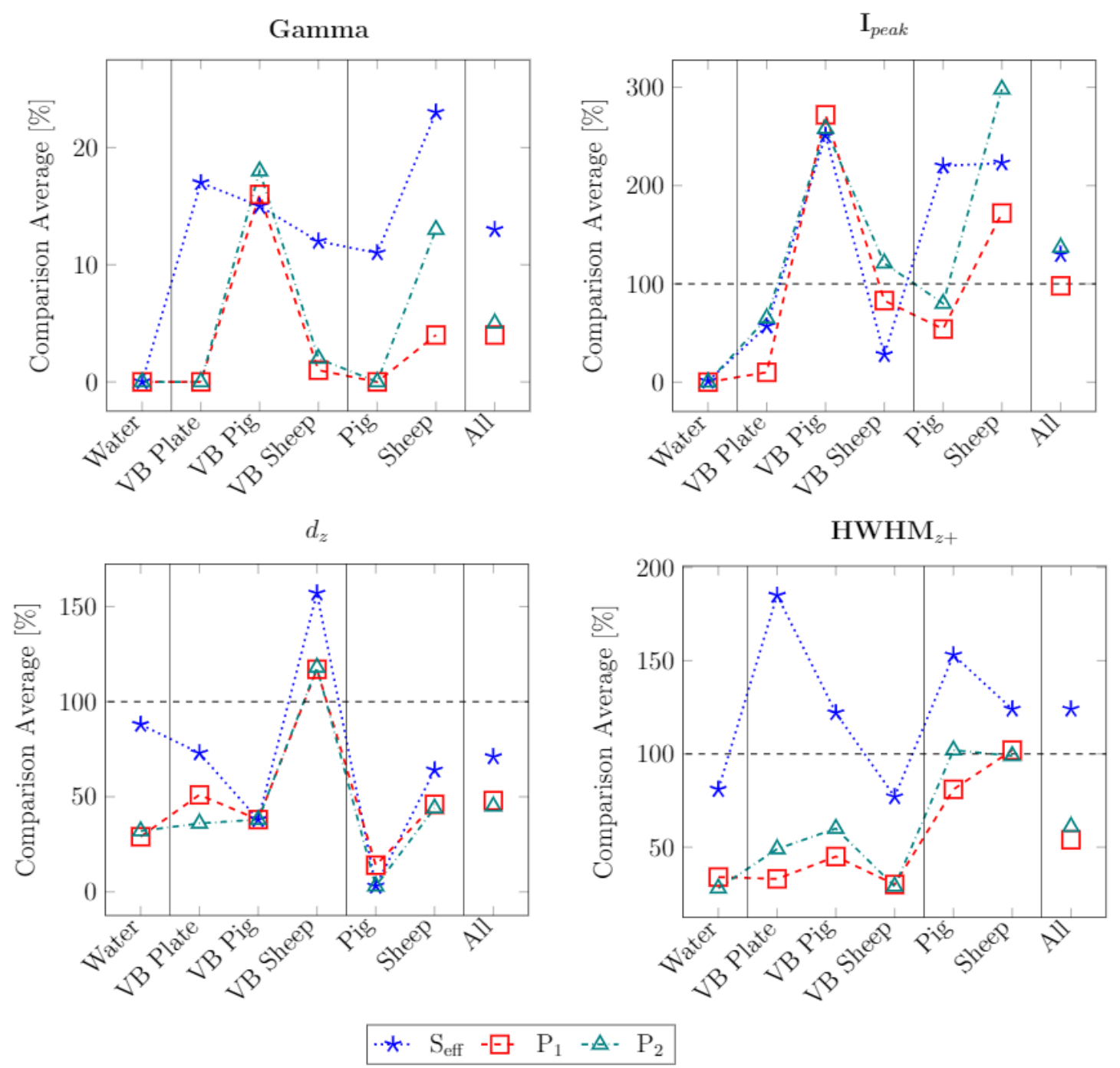

Figure 6: Averaged deviations for each obstacle at different positions between the measured and simulated distributions for the different transducer models: $\mathrm{S}_{\text {eff }}$ and $\mathrm{P}_{1-2}$. Values normalized to the prescribed spatial $(5 \mathrm{~mm})$ and intensity tolerances $(15 \%)$. Values above $100 \%$ fall outside the permitted tolerance. The data in the abscissa are in arbitrary order, so that the dotted lines do not indicate any trend, but are used for a better visual grouping of the results of the different transducers. 
'cosine' and the 'spherical' aperture function simulations. It does, however, also display a pronounced central maximum, similar to that observed with the 'linear' aperture function. The radial dependence of phase primarily reflects the varying path length from the piezo element to the transducer surface. All aperture functions result in similar phase patterns. In general, it can be seen that a pressure boundary condition with suitable aperture function is capable of reproducing the physical exposure, even though a velocity boundary condition might be more physically meaningful and can produce noticeable differences, particularly with regard to secondary maxima near the piezo-element.

The backpropagated pressure distribution on the plane encompassing the transducer aperture was used as source in simulations with and without sheep skull obstacle. Table 4 quantifies the obtained agreement with the measured intensity distribution. While the agreement in water is high, some differences are obtained in the presence of a skull (reflected in all of the metrics, see Table A.8 and Figure 5). In particular, a large shift of the beam position occurs in that case. The disagreement metrics are larger than those observed for the physical transducer simulation models, but smaller than those obtained using $\mathrm{S}_{\text {eff }}$. These differences could be due to limitations of the backpropagated source approach in handling multiple reflections or uncertainties in the measurements.

A)

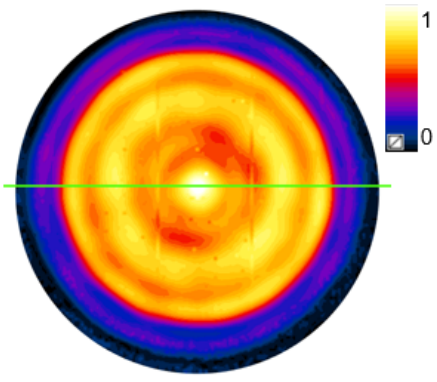

$\|\mathbf{p}\|$

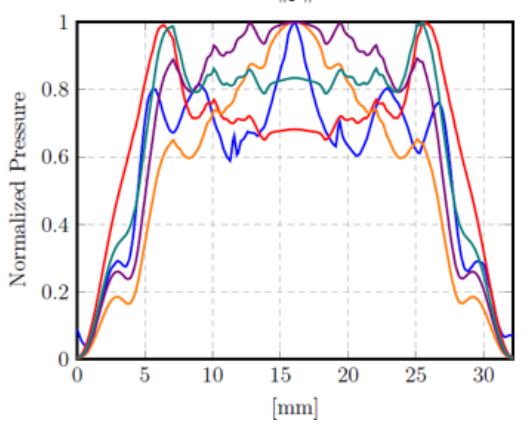

B)
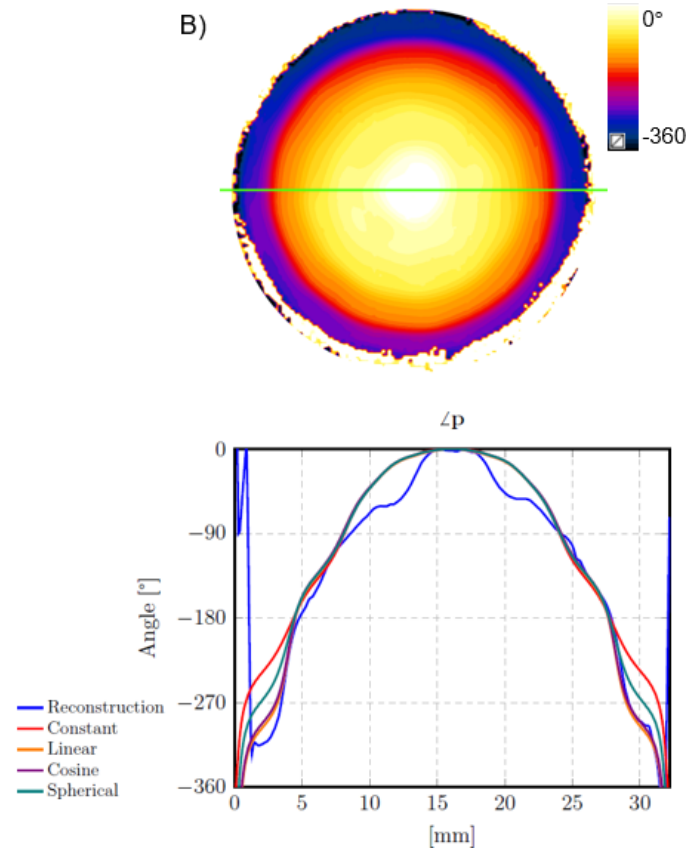

Figure 7: Top: (A) Absolute value and (B) angle of the reconstructed complex pressure at the curved surface of the transducer lens via backpropagation of measurements. Bottom: Line plots of different 'aperture' functions and the reconstruction, interpolated on an arc going through the center of the transducer's curve (green line in top figures).

\subsection{Human head models}

The transcranial intensity distribution results obtained using the human head models can be seen in Figure 8. The different transducer models primarily affect the intensity scaling 
(skull transmission efficacy). The distribution is less affected.

\subsection{Impact of non-linearity and pressure wave approximation}

The WLE simulations of the sheep skull showed no non-linearity impact (differences $<1 \%$ of the peak intensity) at the simulated exposure strength (tested by using WLE with and without non-linear term; in agreement with [28]). The negligible differences $(<1 \%$ of the peak pressure) between the pressure fields obtained using the LAPWE solver and WLE solver without non-linear term can be readily explained by the differing stencil choices and time-integration schemes (higher order for the WLE solver).

\section{Discussion}

Translatability of transducer model. While it is correct that the physical transducer model has been optimized to fit measurements, it is important to note that this optimization was only performed for the water measurements and the fitted model was subsequently used without further adaptation for all the different obstacle setups (bone and Veroblack obstacles).

'Effective' and physical transducer model. The results demonstrate that using the measured transducer surface geometry as pressure source fails to correctly predict the intensity distribution and results in strong deviations in focus location and shape. Applying the 'effective' model geometry provided by the manufacturer or artificially varying the curvature, as frequently done [20,11, 21, 22, 23], permits to mimic focus location, but results in important deviations in focus shape, both in terms of primary focus size and secondary foci in the near-field. Furthermore, the fact that this model is an unphysical model means that it is an 'effective' model only in the absence of obstacles. As the overall intensity distribution provided by the 'effective' model deviates substantially from that of the real transducer, the presence of the obstacle results in a completely different interference pattern that prevents reliable modeling even of focus location (see Figure 4). In contrast, the simulations based on the physical transducer model were substantially more accurate (see Figure 6) for all tested cases after the model was calibrated to fit the measurements obtained for the pure water background. As such, similarly to the 'effective' model, our proposed approach for setting up the physical transducer model requires initial reference measurements, but subsequently results in more accurate predictions of the acoustic beam. However, we only varied the radius of curvature to find the best $S_{\text {eff }}$ (see Section 2.7). A better agreement with the measurements might be obtained when using a more complicated effective model, optimizing for additional free parameters, such as the aperture diameter and the focal distance. Accurate transducer modeling is also expected to be a prerequisite for simulation-driven design of acoustic lenses placed on top of transducers, as in [38, 39].

Transducer modeling in water. Different parameters of the physical transducer model had specific, distinguishable impacts on the intensity distribution (see Figures A.11 and A.12). Changing the attenuation parameters (of the acoustic lens material or the obstacle) hardly affected the intensity distribution and only resulted in a change in magnitude scale. Both 

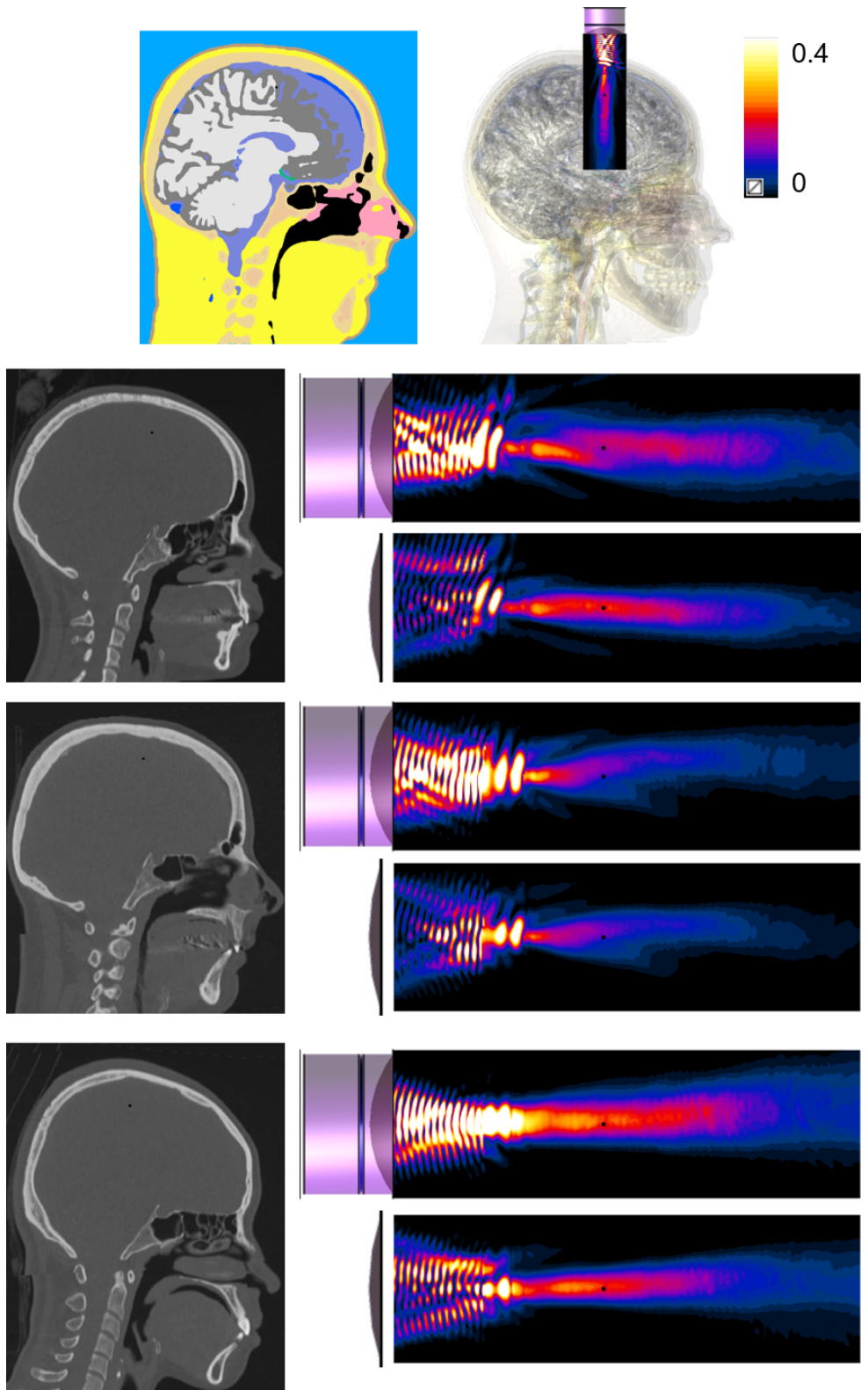

Figure 8: Transcranial intensity (normalized to water) of three different human head models with two different transducer models $\left(\mathrm{P}_{1}\right.$ and $\left.\mathrm{S}_{\text {eff }}\right)$. Top: Sample CT tissue segmentation used to generate head models and simulation setup with superimposed intensity profile. Left: Head models with superimposed point for reference (target location at $50 \mathrm{~mm}$ distance from base of transducers). Right: Resulting normalized intensity beam for the shown transducer models. All colorbars are set to the same normalized intensities for ease of comparison. 
modification of the lens material speed-of-sound and of the piezo element depth have a similar effect, namely a noticeable impact on the intensity distribution. This is due to the fact that a change in speed-of-sound in the region where the wave is still mostly traveling paraxially corresponds to an effective change in the traveled distance. The curved surface primarily produces a radial distance dependent phase delay, which is slightly affected by the speed-of-sound. The mechanical construction of the transducer (e.g., transducer walls and housing) affect the vibrational modes. We have considered the impact of such an effect on the acoustic pressure wave by introducing an aperture function. A change in aperture function has a small impact on focus sharpness, but its main effect is to modify the location and occurrence of secondary foci.

We have modeled the piezo element as time-harmonic Dirichlet boundary condition (prescribed pressure). This is the natural and common choice when using FDTD. However, other methods exist where boundary conditions are commonly defined in terms of prescribed velocities. Constant velocity and constant pressure are not equivalent, as evident, e.g., when looking at the analytical solution for a vibrating circular disk obtained using RayleighSommerfeld integrals [12] where velocity is constant across the transducer surface, while pressure is not. However, this should be investigated further as the different boundary conditions will give rise to different intensity distributions. Replacing the 'hard' with a 'soft' boundary condition modifies the exposure in a similar way as shifting the piezo-depth does (i.e., less pronounced secondary peaks in the near-field, sharper focus). This can be understood as a result of the modified internal interference pattern inside the transducer. In the absence of knowledge about the exact internal mechanical structure of the transducer, the associated modeling ambiguity cannot be avoided.

Sensitivity and tolerances. In line with [26], our results indicate that small variations in acoustic material properties such as speed-of-sound, can have an important impact on the complex interference pattern of (curved) acoustic transducers. Therefore, it is important to reduce uncertainty by properly characterizing the acoustic properties of relevant materials in the sonication setup.

The choice of the tolerances $\left(\Delta d_{x, y, z}\right.$ and $\left.\Delta D\right)$ for the Gamma metric - which is used in this study to judge simulation-measurement agreement - is driven by application specific criteria. That is, prediction errors above the tolerances would significantly compromise the accuracy and precision of the conclusions with regards to the targeted position in the brain and the intensity at the target. This approach is unlike that followed in [26] where agreement tolerances were based on a thorough uncertainty analysis. The Gamma tolerances obtained in [26] at similar frequencies $(550 \mathrm{kHz}$ rather than $500 \mathrm{kHz})$ through uncertainty analysis of sonication in the absence of an obstacle (1.3 times the wavelength in water, i.e. $4 \mathrm{~mm}$; $14 \%$ of the peak intensity) are comparable to the ones used in this study ( $5 \mathrm{~mm}, 15 \%)$. However, a similar uncertainty assessment in the presence of skull obstacles would have resulted in much larger tolerances. Therefore, the approach chosen for this paper results in much stricter criteria that are hard to meet but reflect application needs.

Standing waves in the obstacle. The large difference in acoustic impedance between Veroblack and water (see Table 1 ) results in strong reflections at the interfaces (32\% of the pressure 
amplitude for a plane wave with normal incidence). This leads to a standing wave effect within the obstacle [40], which results in resonator behavior (similar to that known from Fabry-Pérot resonators in laser-physics [41]) and is known to be associated with fluctuations in transmitted power as a function of the effective cavity length (see Figure 9). When the planar Veroblack slab is placed at the focus location, varying the frequency (or equivalently the wavelength or obstacle thickness) results in up to $20 \%$ changes in pressure transmission (>40\% change in intensity, see Figure 9). This standing-wave effect helps explain the focus intensity differences observed with Veroblack obstacles (see Section 3.3). Further research should be performed to assess the relevance of such findings for transcranial sonication. It is expected that skull heterogeneity and losses related to absorption or scattering reduce the occurrence of standing waves. On the other hand, the higher density and speed-of-sound of cortical bone compared to Veroblack - and the related increase in acoustic impedance mismatch - leads to a more than fourfold variability of the transmitted intensity for relatively moderate changes in speed-of-sound assignment and skull thickness modeling (computed analytically for a homogeneous cortical bone plate of realistic thickness immersed in water; see Figure 9). This results in a high sensitivity to modeling errors. This phenomenon has been discussed by [42], where an approach to optimize transcranial focal-gain by suitably tuning the sonication frequency is proposed.

Standing waves between the transducer and the obstacle. Similarly, standing waves occur between the transducer and the obstacle. Changing the transducer models affects this standing-wave configuration - as evident when comparing the 'effective' with the physical transducer model (see Fig. 8) -, which in turn affects the skull exposure. This is likely to be an important factor in explaining the observed changes in the pressure distribution.

Limitations and further work. A 'first principles' approach would consist in performing proper mechanical modeling (i.e., full dynamic stress-strain simulation) of the transducer, from which the vibrational mode would emerge and might allow for accurate predictions of the acoustic beam without the need for reference measurements. However, this is beyond the scope of this paper.

The simulated sonication intensities for the bone skull samples agreed satisfactorily with the measurements only after resorting to species-specific skull attenuation maps (see Table 2). This might reflect different bone compositions between species, which can result in different properties for similar $\mathrm{HU}$ values. A more detailed evaluation of CT image-based modeling of skull properties is the subject of a companion paper [31].

A recent work [43] proposed an alternative way of constructing an ultrasound source model, based on holographic projections. Briefly, this method allows the derivation of an equivalent source model from pressure measurements in a plane parallel to the transducer face by iterative optimization of the pressure distribution on the surface of the equivalent source. Similar to the source reconstructed by back-propagation here, the equivalent source can then be employed in a full-wave model to estimate the ultrasound beam through complex and heterogeneous media. In general, both our and their [43] work highlight the need for source models that are more accurate than 'effective' models. In contrast to our physical 

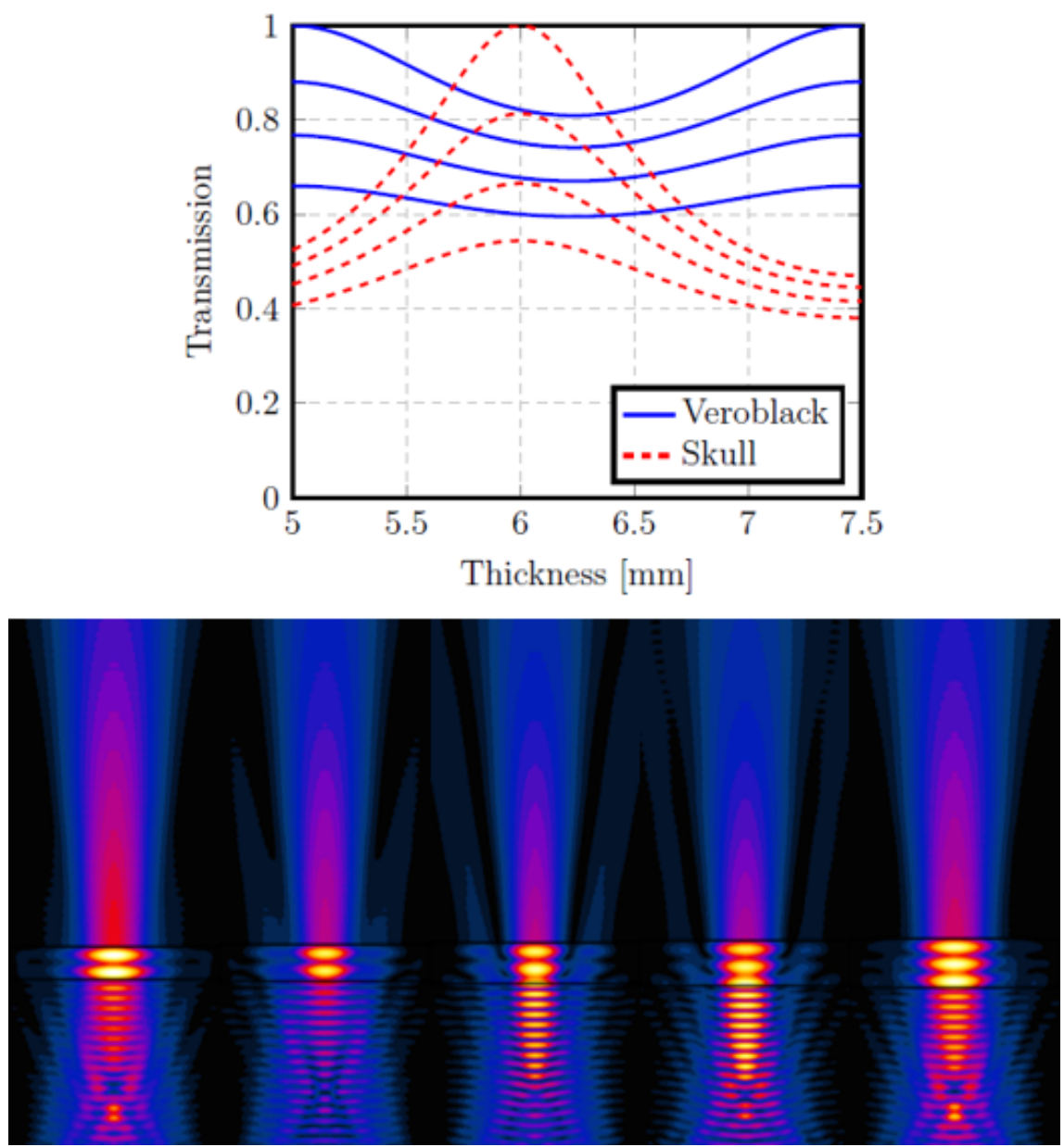

Figure 9: Impact of the standing wave effect on transmission. (Top) Analytically computed plane wave (pressure) transmission factor past a flat, homogeneous obstacle (blue: Veroblack, red: cortical bone $c$ and $\rho$; see Table 1), as a function of obstacle thickness and for different attenuation factors (0, 10, 20, or $30 \%$ attenuation when propagating through the plate). (Bottom) Pressure distribution obtained for a physical transducer model past a Veroblack obstacle of varying thickness $(5-7.5 \mathrm{~mm}$, corresponding to $1-1.5$ wavelengths in the obstacle, $0.5 \mathrm{~mm}$ steps). The color-bar remains identical. Notice that the top figure is computed for plane-wave exposure, while the bottom figure shows results for a focused sonication. 
transducer models, the method in [43] has the advantage of not requiring information about the internal structure of the transducer (nor the construction of a corresponding acoustomechanical model), but depends on measurement data (phases and amplitudes) at very high resolution, accuracy and precision instead, as measurement errors might be amplified in the equivalent source model reconstruction process. Our approach constrains the fitting of the equivalent model to a few key parameters, so that measurement errors are more likely to be identifiable as mismatches between modeled and measured beams.

\section{Conclusions}

Careful transducer modeling and experimental validation is crucial for the reliable simulation of TUS fields. The currently commonly employed approaches - i.e., assigning a boundary condition to the real shape of the transducer surface, or using an 'effective' transducer shape model that has been constructed to produce a focus at the right location in a homogeneous water setup - are inadequate for flat piston transducers with a curved acoustic lens and, possibly, for other geometrically complex transducer models. This is particularly true in the presence of acoustic obstacles and inhomogeneity. This suggests that effective transducer models should be validated also in the presence of complex and inhomogeneous obstacles in order to ensure that they perform well also in realistic usage scenarios. Even physics-based transducer modeling can sometimes fail to reach the chosen, clinically motivated, agreement criteria ( $15 \%$ peak intensity, $5 \mathrm{~mm}$ for focus position and length). An optimal, but highly demanding and typically impracticable approach would include complete mechanical modeling of the transducer with its housing and fixation. However, a compromise combining improved acoustic modeling of the transducer and its internal structure with an aperture function to account for the missing mechanical modes can be an acceptable solution, but requires the acquisition of reference data using hydrophone measurements. An alternative approach would be to adapt the transducer model or the transducer placement heuristically, using image-based information, to compensate for, e.g., the skull lens effect [44]. Image-based information, such as MRI data, is readily available and can also be used to optimize the sonication path.

If possible, experimental effort should be invested in characterizing sensitive material properties of the transducer components and obstacle media (particularly speed-of-sound and species-specific attenuation). Comprehensive uncertainty assessment should typically be performed along with computational modeling. Standing wave effects have been found to have a high impact on the sensitivity and accuracy of transmitted intensity predictions for the 3D-printed and other homogeneous obstacles (up to fourfold variation of peak transcranial intensity for relatively small speed-of-sound assignment errors in cortical skull). Additional studies should be performed to investigate how much skull heterogeneity affects the formation of standing-waves. Approaches involving, e.g., stochastic modulation, to suppress standing waves have been proposed [45, 46, 47]. 


\section{Acknowledgements}

CP was supported by a PhD stipend of the Technical University of Denmark. AT was supported by the Lundbeck Foundation (grant no. R118-A11308). AT and HRS were supported by a synergy grant from the NovoNordisk Foundation (Interdisciplinary Synergy Program 2014; grant number NNF14OC0011413). HRS holds a professorship in Precision Medicine at the Institute of Clinical Medicine, Faculty of Health and Medical Sciences, Copenhagen University, sponsored by the Lundbeck Foundation. 
Appendix A. Results Tables and Figures 


\begin{tabular}{llrrrrrr}
\hline \multicolumn{7}{c}{ Veroblack Plate Comparison [\%] } \\
\hline & & Gamma & $\mathrm{I}_{p e a k}$ & \multicolumn{1}{c}{$d_{z}$} & \multicolumn{1}{c}{$d$} & FWHM $_{z}$ & HWHM $_{z+}$ \\
\hline VB Plate & $\mathrm{S}_{\text {eff }}$ & 18.9 & -77.1 & -134.9 & 134.9 & -220.6 & -180.6 \\
(2 cm dist.) & $\mathrm{P}_{1}$ & 0.0 & 5.4 & -71.9 & 71.9 & -57.6 & -55.1 \\
& $\mathrm{P}_{2}$ & 0.0 & -39.6 & -41.9 & 41.9 & -97.5 & -51.6 \\
\hline VB Plate & $\mathrm{S}_{\text {eff }}$ & 14.9 & -37.4 & -11.5 & 18.9 & - & -190.3 \\
$\mathbf{( 3 ~ c m ~ d i s t . ) ~}$ & $\mathrm{P}_{1}$ & 0.0 & -15.4 & -29.6 & 33.9 & - & -10.8 \\
& $\mathrm{P}_{2}$ & 0.0 & -89.8 & -29.6 & 33.9 & - & 46.2 \\
\hline
\end{tabular}

Table A.5: Comparison of the difference metrics of simulated and measured intensity distributions with the Veroblack plate obstacles at varying positions (the tolerance-normalized deviation in \%). 


\begin{tabular}{llrrr}
\hline \multicolumn{4}{c}{ Veroblack Printed Skulls } & \multicolumn{3}{c}{ Comparison [\%] } \\
\hline & & Gamma & \multicolumn{1}{c}{$\mathrm{I}_{\text {peak }}$} & HWHM $_{z+}$ \\
\hline VB Pig 1 & $\mathrm{S}_{\text {eff }}$ & 25.4 & -284.4 & -65.1 \\
& $\mathrm{P}_{1}$ & 0.0 & 100.5 & -57.0 \\
& $\mathrm{P}_{2}$ & 0.0 & 71.1 & -41.0 \\
\hline VB Pig 2A & $\mathrm{S}_{\text {eff }}$ & 47.7 & -530.0 & -161.5 \\
& $\mathrm{P}_{1}$ & 0.0 & -74.7 & 26.5 \\
& $\mathrm{P}_{2}$ & 0.0 & -67.5 & 26.5 \\
\hline VB Pig 2B & $\mathrm{S}_{\text {eff }}$ & 0.0 & 118.7 & -127.2 \\
& $\mathrm{P}_{1}$ & 44.0 & -616.4 & -72.3 \\
& $\mathrm{P}_{2}$ & 48.4 & -616.4 & -72.3 \\
\hline VB Pig 2C & $\mathrm{S}_{\text {eff }}$ & 0.1 & 287.6 & -155.8 \\
& $\mathrm{P}_{1}$ & 8.0 & -113.1 & -35.9 \\
& $\mathrm{P}_{2}$ & 5.6 & 12.2 & -120.9 \\
\hline VB Pig 3 & $\mathrm{S}_{\text {eff }}$ & 0.0 & -32.3 & -102.5 \\
& $\mathrm{P}_{1}$ & 27.6 & -454.9 & -35.2 \\
& $\mathrm{P}_{2}$ & 35.5 & -524.4 & -40.2 \\
\hline
\end{tabular}

Table A.6: Comparison of the difference metrics of simulated and measured intensity distributions with the pig-skull-shaped Veroblack obstacle at varying positions (the tolerance-normalized deviation in \%).

\begin{tabular}{llrrrrr}
\hline \multicolumn{6}{c}{ Veroblack Printed Skulls Comparison [\%] } \\
\hline VB Sheep 1 & $\mathrm{S}_{\text {eff }}$ & 3.9 & -17.4 & -99.7 & 101.1 & -80.6 \\
& $\mathrm{P}_{1}$ & 0.0 & -42.0 & -92.2 & 93.4 & 27.3 \\
& $\mathrm{P}_{2}$ & 4.1 & -151.2 & -94.7 & 95.6 & 0.6 \\
\hline VB Sheep 2A & $\mathrm{S}_{\text {eff }}$ & 1.7 & 30.2 & -125.0 & 126.7 & -70.5 \\
& $\mathrm{P}_{1}$ & 1.8 & 120.3 & -122.2 & 124.2 & -11.3 \\
& $\mathrm{P}_{2}$ & 0.4 & 132.5 & -122.2 & 124.2 & -38.7 \\
\hline VB Sheep 3 & $\mathrm{S}_{\text {eff }}$ & 31.0 & -35.5 & -247.1 & 247.2 & -80.0 \\
& $\mathrm{P}_{1}$ & 2.0 & 85.2 & -136.1 & 137.3 & 50.7 \\
& $\mathrm{P}_{2}$ & 0.7 & 80.1 & -136.1 & 137.6 & 48.3 \\
\hline
\end{tabular}

Table A.7: Comparison of the difference metrics of simulated and measured intensity distributions with the sheep-skull-shaped Veroblack obstacle at varying positions (the tolerance-normalized deviation in \%). 


\begin{tabular}{|c|c|c|c|c|c|c|}
\hline \multicolumn{7}{|c|}{ Skulls Comparison [\%] } \\
\hline & & Gamma & $\mathrm{I}_{\text {peak }}$ & $d_{z}$ & $d$ & $\mathrm{HWHM}_{z+}$ \\
\hline \multirow[t]{3}{*}{ Pig 1} & $\mathrm{~S}_{\text {eff }}$ & 16.4 & -134.7 & 3.4 & 30.3 & -174.9 \\
\hline & $\mathrm{P}_{1}$ & 0.0 & -11.7 & 10.6 & 33.7 & -53.0 \\
\hline & $\mathrm{P}_{2}$ & 0.1 & -55.6 & 2.2 & 31.9 & -73.3 \\
\hline \multirow[t]{3}{*}{ Pig 2A } & $\mathrm{S}_{\mathrm{eff}}$ & 6.3 & -238.6 & - & - & -46.5 \\
\hline & $\mathrm{P}_{1}$ & 0.0 & -50.5 & - & - & 67.2 \\
\hline & $\mathrm{P}_{2}$ & 0.1 & -129.5 & - & - & 62.3 \\
\hline \multirow[t]{3}{*}{ Pig 2B } & $\mathrm{S}_{\mathrm{eff}}$ & 21.8 & -53.6 & - & - & -187.1 \\
\hline & $\mathrm{P}_{1}$ & 0.0 & 19.1 & - & - & -30.0 \\
\hline & $\mathrm{P}_{2}$ & 0.4 & -19.6 & - & - & -54.9 \\
\hline \multirow[t]{3}{*}{ Pig 2C } & $\mathrm{S}_{\mathrm{eff}}$ & 6.8 & - & - & - & 212.1 \\
\hline & $\mathrm{P}_{1}$ & 0.3 & - & - & - & 44.9 \\
\hline & $\mathrm{P}_{2}$ & 0.4 & - & - & - & 82.3 \\
\hline \multirow[t]{3}{*}{ Pig 3} & $S_{\text {eff }}$ & 4.2 & -193.4 & - & - & 142.1 \\
\hline & $\mathrm{P}_{1}$ & 0.2 & -36.1 & - & - & 209.4 \\
\hline & $\mathrm{P}_{2}$ & 0.7 & -108.6 & - & - & 236.9 \\
\hline \multirow[t]{5}{*}{ Sheep 1} & $\mathrm{~S}_{\mathrm{eff}}$ & 38.7 & -105.2 & -91.0 & 101.6 & -123.2 \\
\hline & $\mathrm{P}_{1}$ & 4.2 & 25.2 & -27.5 & 48.7 & 3.9 \\
\hline & $\mathrm{P}_{2}$ & 11.4 & 75.9 & -28.7 & 50.4 & -60.7 \\
\hline & $\mathrm{M}_{\mathrm{B}-\mathrm{P}}$ & 15.9 & 49.6 & -81.4 & 90.4 & -87.2 \\
\hline & $\mathrm{P}_{1-\mathrm{W} 1492}$ & 6.4 & 35.7 & -190 & 192 & 110.5 \\
\hline \multirow[t]{3}{*}{ Sheep 2A } & $\mathrm{S}_{\mathrm{eff}}$ & 7.9 & -115.8 & 98.6 & 101 & -241.1 \\
\hline & $\mathrm{P}_{1}$ & 3.8 & -192.4 & 106.5 & 110.5 & -16.2 \\
\hline & $\mathrm{P}_{2}$ & 7.6 & -256.1 & 102.8 & 105.2 & -33.5 \\
\hline \multirow[t]{3}{*}{ Sheep 3} & $\mathrm{~S}_{\mathrm{eff}}$ & 21.6 & -447.1 & 1.7 & 32 & -36.9 \\
\hline & $\mathrm{P}_{1}$ & 4.8 & -297.2 & 5.4 & 31 & 286.3 \\
\hline & $\mathrm{P}_{2}$ & 19.4 & -5.6 & 1.7 & 31 & 231.4 \\
\hline
\end{tabular}

Table A.8: Comparison of the difference metrics of simulated and measured intensity distributions with the real skull obstacles at varying positions (the tolerance-normalized deviation in \%). $d_{z}, d$ and $\mathrm{I}_{\text {peak }}$ comparisons omitted for simulation results where maxima occurs at first measurement position. $\mathrm{P}_{1-\mathrm{W} 1492}$ indicates the simulation where the acoustic property of water at $20^{\circ} \mathrm{C}$ were employed. 

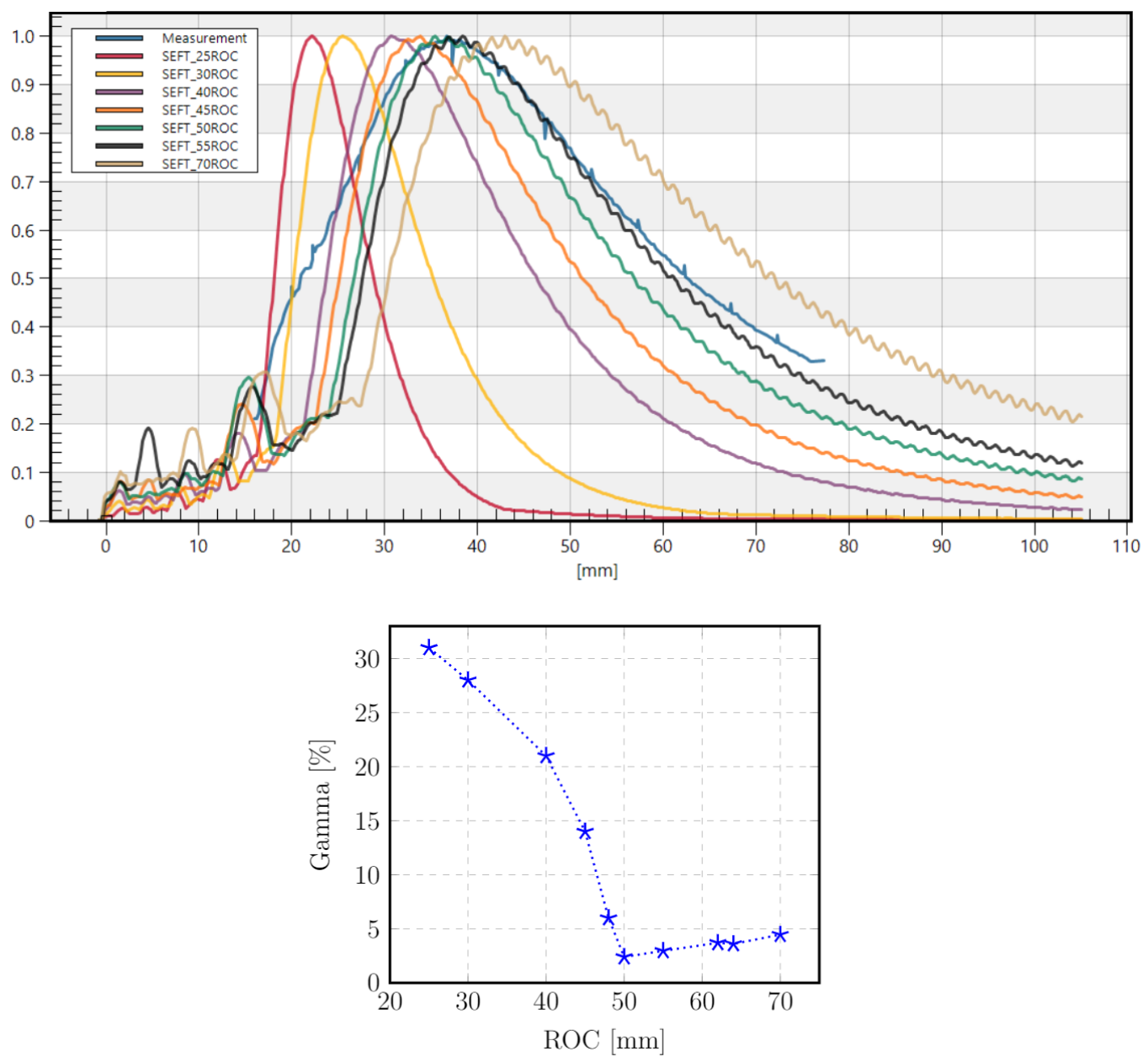

Figure A.10: Top: the intensity profiles of the measurement and the simplified SEFT transducer models with different radii of curvature (ROC) normalized by the maximum. The x-axis indicates the distance from the base of the transducer in the longitudinal direction. Bottom: The Gamma metric comparing the disagreement of the measurement and the transducer models with different ROC is shown. Notice that the model with $50 \mathrm{ROC}$ is the best match. We employed the latter throughout the paper and called it $\mathrm{S}_{\mathrm{eff}}$. 

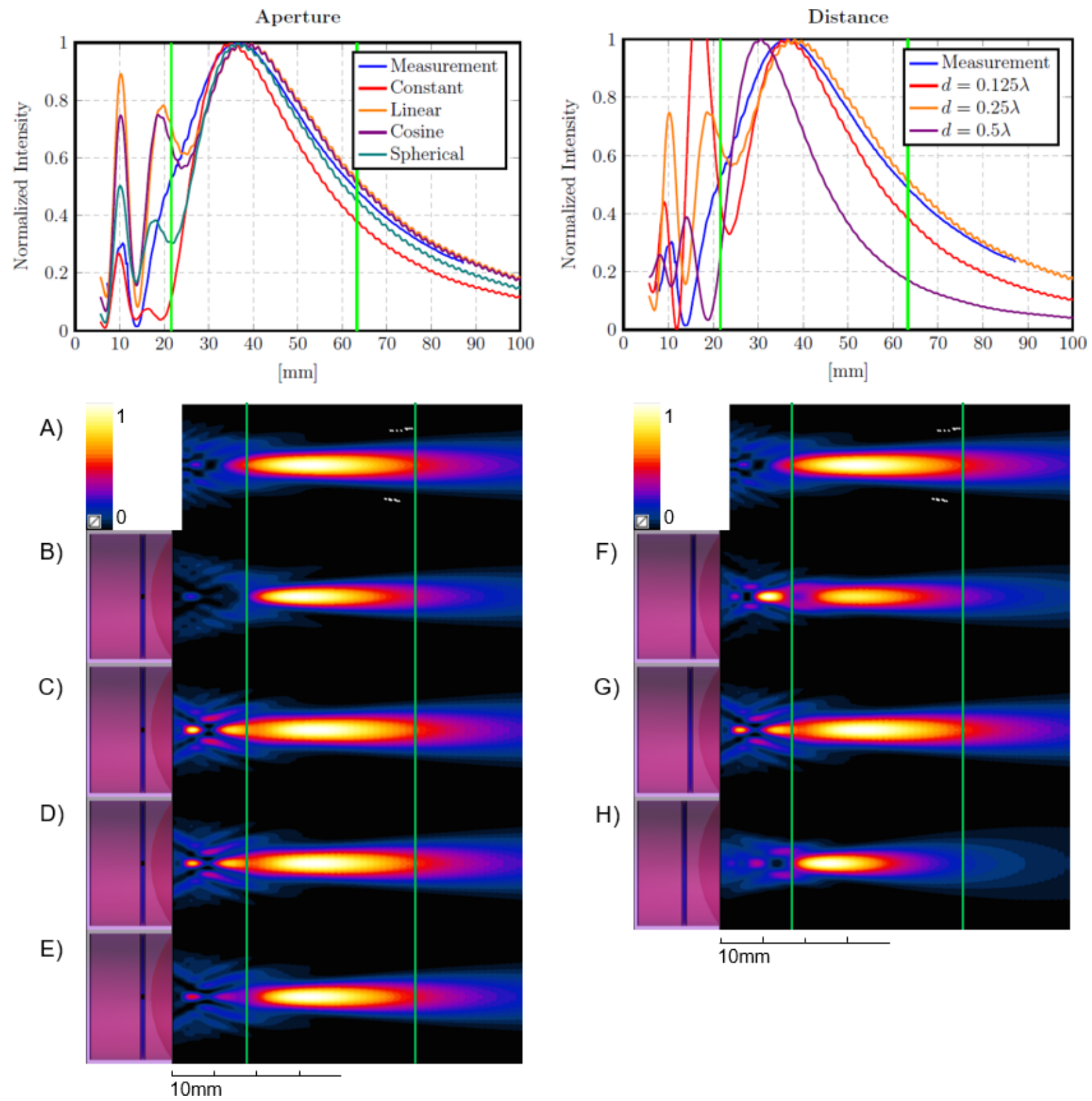

F)
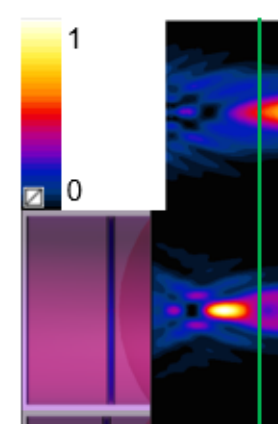

G)
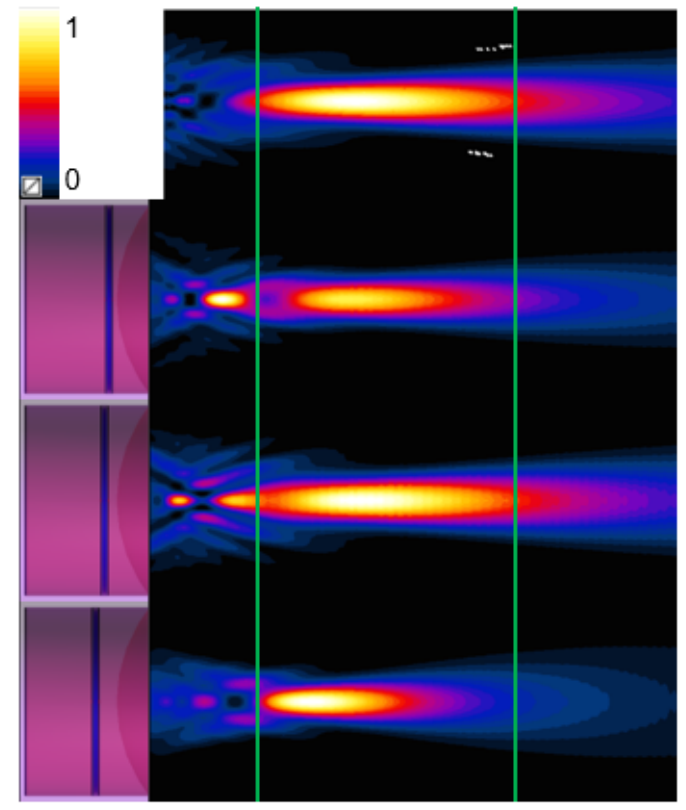

$10 \mathrm{~mm}$

Figure A.11: Variation of the normalized intensity distribution of a physical transducer model resulting from changes: (B-E) to the aperture function (radial pressure variation of the piezo element boundary condition; constant, linear, cosine, and spherical, respectively), and (F-H) to the acoustic lens layer thickness $(0.125$, 0.25 , and 0.5 wavelengths). (A) The measurement reference. (Top) Plot along the symmetry axis. 

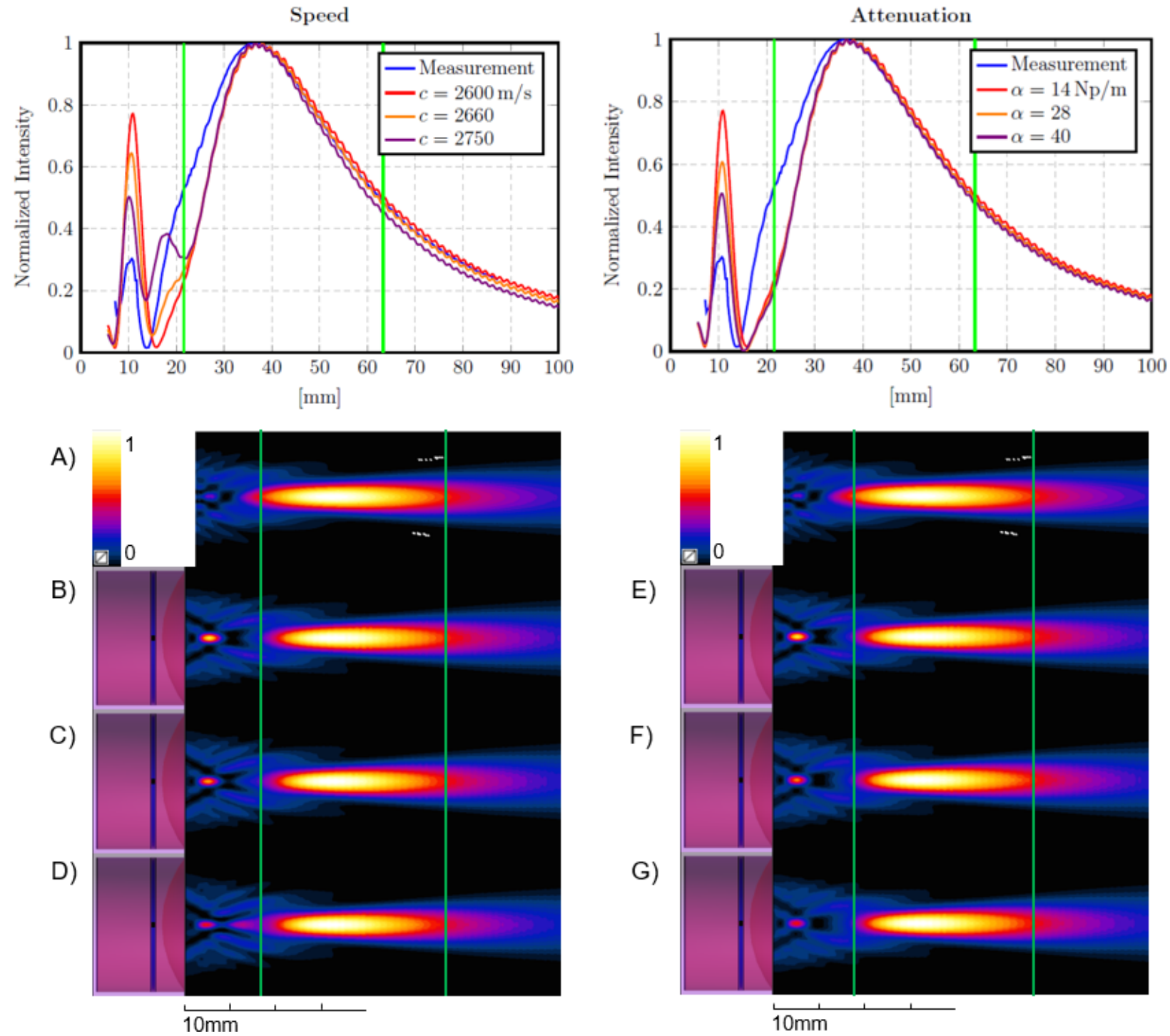

E)
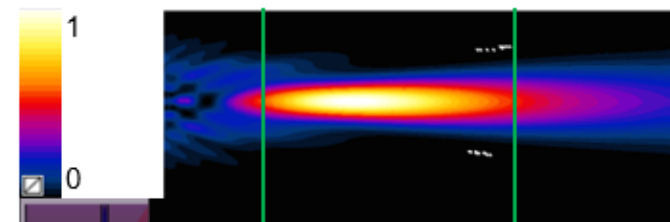

F)

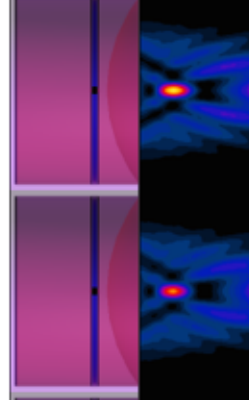

G)

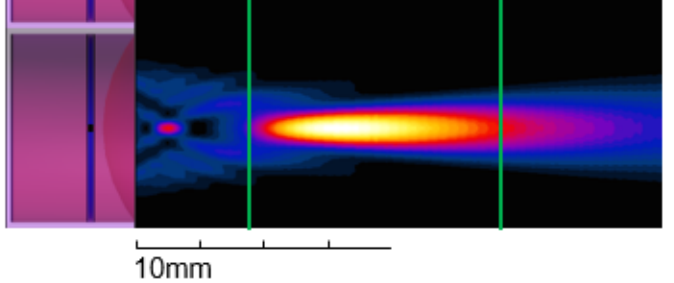

Figure A.12: Variation of the normalized intensity distribution of a physical transducer model resulting from changes: (B-D) to the speed-of-sound of the acrylic acoustic lens (2600, 2660, and $2750 \mathrm{~m} / \mathrm{s}$ ), and (E-G) to the acrylic acoustic lens attenuation $(14,28$, and $40 \mathrm{~Np} / \mathrm{m})$. (A) The measurement reference. (Top) Plot along the symmetry axis. 


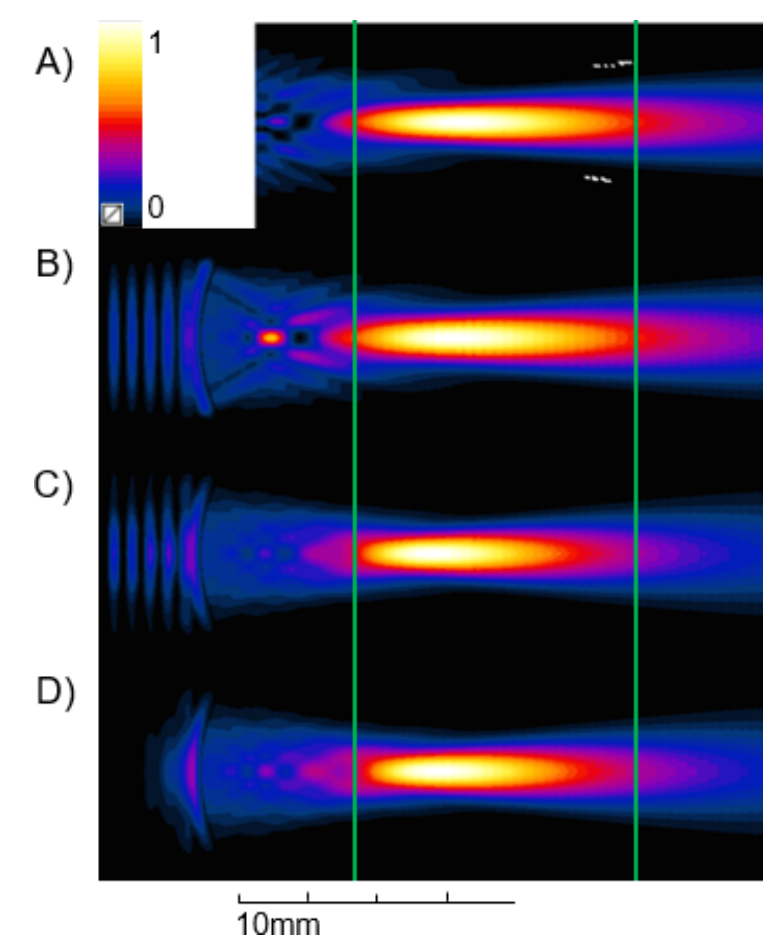

Figure A.13: Comparison between piezo-element boundary conditions in simulations of the beam profile in a pure water background. (A) Reference measurement. Normalized intensity distribution with piezo (B) 'hard' source and (C-D) 'soft' source. The casing is treated as a perfect reflector. The backing layer is modeled with the same density and speed-of-sound as the acoustic lens and is assigned varying absorption values (B-C: $50 \mathrm{~Np} / \mathrm{m}$ and A: $150 \mathrm{~Np} / \mathrm{m}$ ). The green line is provided as reference to indicate the extent of the FWHM in the measurement. 


\section{References}

[1] Alexander Bystritsky, Alex S. Korb, Pamela K. Douglas, Mark S. Cohen, William P. Melega, Amit P. Mulgaonkar, Antonio Desalles, Byoung Kyong Min, and Seung Schik Yoo. A review of low-intensity focused ultrasound pulsation, jul 2011.

[2] Yusuf Tufail, Alexei Matyushov, Nathan Baldwin, Monica L. Tauchmann, Joseph Georges, Anna Yoshihiro, Stephen I Helms Tillery, and William J. Tyler. Transcranial pulsed ultrasound stimulates intact brain circuits. Neuron, 66(5):681-94, 2010.

[3] Seung Schik Yoo, Alexander Bystritsky, Jong Hwan Lee, Yongzhi Zhang, Krisztina Fischer, Byoung Kyong Min, Nathan J. McDannold, Alvaro Pascual-Leone, and Ferenc A. Jolesz. Focused ultrasound modulates region-specific brain activity. NeuroImage, 56(3):1267-1275, 2011.

[4] Thomas Deffieux, Youliana Younan, Nicolas Wattiez, Mickael Tanter, Pierre Pouget, and Jean François Aubry. Low-intensity focused ultrasound modulates monkey visuomotor behavior. Current Biology, 23(23):2430-2433, 2013.

[5] Wonhye Lee, Stephanie D. Lee, Michael Y. Park, Lori Foley, Erin Purcell-Estabrook, Hyungmin Kim, Krisztina Fischer, Lee So Maeng, and Seung Schik Yoo. Image-Guided Focused Ultrasound-Mediated Regional Brain Stimulation in Sheep. Ultrasound in Medicine and Biology, 42(2):459-470, 2016.

[6] Dianne Daniels, Shirley Sharabi, David Last, David Guez, Sharona Salomon, Zion Zivli, David Castel, Alex Volovick, Javier Grinfeld, Itay Rachmilevich, Talia Amar, Sigal Liraz-Zaltsman, Narek Sargsyan, Yael Mardor, and Sagi Harnof. Focused Ultrasound-Induced Suppression of Auditory Evoked Potentials in Vivo. Ultrasound in Medicine and Biology, 44(5):1022-1030, 2018.

[7] Wonhye Lee, Hyungmin Kim, Yujin Jung, In Uk Song, Yong An Chung, and Seung Schik Yoo. Imageguided transcranial focused ultrasound stimulates human primary somatosensory cortex. Scientific Reports, 5:8743, 2015.

[8] Wonhye Lee, Hyun Chul Kim, Yujin Jung, Yong An Chung, In Uk Song, Jong Hwan Lee, and Seung Schik Yoo. Transcranial focused ultrasound stimulation of human primary visual cortex. Scientific Reports, 6(1):34026, 2016.

[9] Wonhye Lee, Yong An Chung, Yujin Jung, In Uk Song, and Seung Schik Yoo. Simultaneous acoustic stimulation of human primary and secondary somatosensory cortices using transcranial focused ultrasound. BMC Neuroscience, 17(1):68, 2016.

[10] Wynn Legon, Tomokazu F. Sato, Alexander Opitz, Jerel Mueller, Aaron Barbour, Amanda Williams, and William J. Tyler. Transcranial focused ultrasound modulates the activity of primary somatosensory cortex in humans. Nature Neuroscience, 17(2):322-329, 2014.

[11] James Robertson, Eleanor Martin, Ben Cox, and Bradley E. Treeby. Sensitivity of simulated transcranial ultrasound fields to acoustic medium property maps. Physics in Medicine and Biology, 62(7):2559$2580,2017$.

[12] Adamos Kyriakou. Multi-Physics Computational Modeling of Focused Ultrasound Therapies. PhD thesis, ETH Zürich, 2015.

[13] F J Fry and J E Barger. Acoustical properties of the human skull. The Journal of the Acoustical Society of America, 63(5):1576-90, may 1978.

[14] D N White, G R Curry, and R J Stevenson. The acoustic characteristics of the skull. Ultrasound in Medicine and Biology, 4(3):225-252, 1978.

[15] J A Evans and M B Tavakoli. Ultrasonic attenuation and velocity in bone. Physics in Medicine and Biology, 35(10):1387-1396, oct 1990.

[16] J. F. Aubry, M. Tanter, M. Pernot, J.L. Thomas, and M. Fink. Experimental demonstration of noninvasive transskull adaptive focusing based on prior computed tomography scans. The Journal of the Acoustical Society of America, 113(1):84-93, 2003.

[17] Samuel Pichardo, Vivian W. Sin, and Kullervo Hynynen. Multi-frequency characterization of the speed of sound and attenuation coefficient for longitudinal transmission of freshly excised human skulls. Physics in Medicine and Biology, 56(1):219-250, 2011.

[18] Christopher W. Connor, Greg T. Clement, and Kullervo Hynynen. A unified model for the speed 
of sound in cranial bone based on genetic algorithm optimization. Physics in Medicine and Biology, 47(22):3925-3944, 2002.

[19] Hagisonic. Characterization of ultrasonic probe. Technical report, Hagisonic, 2016.

[20] J. L. Teja, S. A. Lopez-Haro, L. Leija, and A. Vera. A finite element simulation of High Intensity Focused Ultrasound with polyacrylamide as coupling material for acoustic hemostasis. Pan American Health Care Exchanges, PAHCE, pages 1-6, 2013.

[21] Jerel K. Mueller, Leo Ai, Priya Bansal, and Wynn Legon. Computational exploration of wave propagation and heating from transcranial focused ultrasound for neuromodulation. Journal of Neural Engineering, 13(5):1-14, 2016.

[22] Matthew Pelekanos, Gerhard Leinenga, Mostafa Odabaee, Maryam Odabaee, Siamak Saifzadeh, Roland Steck, and Jürgen Götz. Establishing sheep as an experimental species to validate ultrasound-mediated blood-brain barrier opening for potential therapeutic interventions. Theranostics, 8(9):2583-2602, 2018.

[23] Eleanor Martin, Yan To Ling, and Bradley E Treeby. Simulating Focused Ultrasound Transducers Using Discrete Sources on Regular Cartesian Grids. IEEE Transactions on Ultrasonics, Ferroelectrics, and Frequency Control, 63(10):1535-1542, 2016.

[24] PA Hasgall, E Neufeld, MC Gosselin, A Klingenböck, and N Kuster. It'is database for thermal and electromagnetic parameters of biological tissues. Version 3.0, 2015.

[25] Alan R Selfridge. Isotropic Materials. Ieee Transactions on Sonics and Ultrasonics, 32(3):381-394, 1985.

[26] Esra Neufeld, Adamos Kyriacou, Wolfgang Kainz, and Niels Kuster. Approach to Validate SimulationBased Distribution Predictions Combining the Gamma-Method and Uncertainty Assessment: Application to Focused Ultrasound. Journal of Verification, Validation and Uncertainty Quantification, 1(3):031006, 2016.

[27] Guillaume Renaud, Samuel Callé, Jean Pierre Remenieras, and Marielle Defontaine. Exploration of trabecular bone nonlinear elasticity using time-of-flight modulation. IEEE Transactions on Ultrasonics, Ferroelectrics, and Frequency Control, 55(7):1497-1507, jul 2008.

[28] Jerel K. Mueller, Leo Ai, Priya Bansal, and Wynn Legon. Numerical evaluation of the skull for human neuromodulation with transcranial focused ultrasound. Journal of Neural Engineering, 14(6), 2017.

[29] M Tanter, M Fink, L Marsac, J-F Aubry, M Pernot, G Montaldo, F Marquet, M Pernot, J-F Aubry, G Montaldo, L Marsac, M Tanter, and M Fink. Non-invasive transcranial ultrasound therapy based on a 3D CT scan: protocol validation and in vitro results. Physics in Medicine and Biology, 54(9):2597-2613, may 2009.

[30] Rokuro Hatakeyama, Masazumi Yoshizawa, and Tadashi Moriya. Method for the measurement of acoustic impedance and speed of sound in a small region of bone using a fused quartz rod as a transmission line. Japanese Journal of Applied Physics, Part 1: Regular Papers and Short Notes and Review Papers, 39(11):6449-6454, 2000.

[31] Hazael Montanaro, Cristina Pasquinelli, Hyunjoo J. Lee, Lars G. Hanson, Niels Kuster, Hartwig R. Siebner, Axel Thielscher, and Esra Neufeld. The effects of ct image parameters and noise on the accuracy of simulations of transcranial focused ultrasound. submitted, 2019.

[32] Michiel Postema. Fundamentals of Medical Ultrasonics. Spon Press, 2018.

[33] Daniel A Low. Gamma dose distribution evaluation tool. In Journal of Physics: Conference Series, volume 250, pages 349-359. IOP Publishing, nov 2010.

[34] Randy L King, Julian R Brown, William T Newsome, and Kim Butts Pauly. Effective parameters for ultrasound-induced in vivo neurostimulation. Ultrasound in Medicine and Biology, 39(2):312-331, 2013.

[35] J. R. Shewell and E Wolf. Inverse Diffraction and a New Reciprocity Theorem*. Journal of the Optical Society of America, 58(12):1596, 1968.

[36] Mark E Schafer and Peter A Lewin. Transducer Characterization Using The Angular Spectrum Method. Journal of the Acoustical Society of America, 85(5):2202-2214, 1989.

[37] Silvia Farcito, Oula Puonti, Hazael Montanaro, Guilherme B. Saturnino, Jesper D. Nielsen, Camilla G. Madsen, Hartwig R. Siebner, Esra Neufeld, Niels Kuster, Bryn A. Lloyd, and Axel Thielscher. Ac- 
curate anatomical head segmentations: a data set for biomedical simulations. In 201941 st Annual International Conference of the IEEE Engineering in Medicine and Biology Society (EMBC), pages 6118-6123. Institute of Electrical and Electronics Engineers (IEEE), oct 2019.

[38] Guillaume Maimbourg, Alexandre Houdouin, Thomas Deffieux, Mickael Tanter, and Jean François Aubry. 3D-printed adaptive acoustic lens as a disruptive technology for transcranial ultrasound therapy using single-element transducers. Physics in Medicine and Biology, 63(2):025026, jan 2018.

[39] Marcelino Ferri, José M. Bravo, Javier Redondo, and Juan V. Sánchez-Pérez. Enhanced Numerical Method for the Design of 3-D-Printed Holographic Acoustic Lenses for Aberration Correction of SingleElement Transcranial Focused Ultrasound. Ultrasound in Medicine and Biology, 45(3):867-884, mar 2019.

[40] Richard SC Cobbold. Foundations of biomedical ultrasound. Oxford university press, 2006.

[41] Nur Ismail, Cristine Calil Kores, Dimitri Geskus, and Markus Pollnau. Fabry-pérot resonator: spectral line shapes, generic and related airy distributions, linewidths, finesses, and performance at low or frequency-dependent reflectivity. Optics express, 24(15):16366-16389, 2016.

[42] P. J. White, G. T. Clement, and K. Hynynen. Local frequency dependence in transcranial ultrasound transmission. In Physics in Medicine 8 Biology, volume 51, page 2293, 2006.

[43] B. Treeby, F. Lucka, E. Martin, and B. T. Cox. Equivalent-Source Acoustic Holography for Projecting Measured Ultrasound Fields Through Complex Media. IEEE Transactions on Ultrasonics, Ferroelectrics, and Frequency Control, 65(10):1857-1864, 2018.

[44] Thomas Deffieux and Elisa Konofagou. Numerical study of a simple transcranial focused ultrasound system applied to blood-brain barrier opening. IEEE Transactions on Ultrasonics, Ferroelectrics, and Frequency Control, 57(12):2637-2653, 2010.

[45] Mohammed A Samoudi, Timothy Van Renterghem, and Dick Botteldooren. Computational modeling of a single-element transcranial focused ultrasound transducer for subthalamic nucleus stimulation. Journal of neural engineering, 16(2):026015, apr 2019.

[46] Patrick Peiyong Ye, Julian R. Brown, and Kim Butts Pauly. Frequency dependence of ultrasound neurostimulation in the mouse brain. Ultrasound in Medicine and Biology, 42(7):1512-1530, jul 2016.

[47] Sai Chun Tang and Gregory T. Clement. Standing-wave suppression for transcranial ultrasound by random modulation. IEEE Transactions on Biomedical Engineering, 57(1):203-205, jan 2010. 